\title{
Propolis microencapsulation by double emulsion solvent evaporation approach: Comparison of different polymeric matrices and extract to polymer ratio
}

\author{
Filipa Paulo $^{a}$, Vanessa Paula ${ }^{b}$, Leticia M. Estevinhoc ${ }^{c}$ Lúcia Santos ${ }^{a, *}$ \\ a LEPABE - Laboratory for Process Engineering, Environment, Biotechnology and Energy, Faculty of Engineering, \\ University of Porto, Rua Dr. Roberto Frias, 4200-465 Porto, Portugal \\ b Instituto Politécnico de Bragança, Campus de Santa Apolónia, 5300-253 Bragança, Portugal \\ c Centro de Investigação de Montanha (CIMO), Instituto Politécnico de Bragança, Campus de Santa Apolónia, \\ 5300-253 Bragança, Portugal
}

\section{A R T I C L E I N F O}

\section{Article history:}

Received 29 June 2020

Received in revised form 11

February 2021

Accepted 28 March 2021

Available online 14 April 2021

\section{Keywords:}

Propolis

Double emulsion

Solvent evaporation

Microencapsulation

Antioxidants

Polyphenols

\begin{abstract}
A B S T R A C T
Propolis, a heterogeneous matrix produced by honeybees, is an antioxidant amongst other valuable biological properties. Nevertheless, the practical application of propolis, especially for the food industry, is still limited due to its relative high hydrophilicity and strong/unpleasant taste and aroma. Accordingly, microencapsulation arises a potential alternative approach to protect propolis compounds while allowing to mask its unpleasant sensory properties.

The purpose of this study was to microencapsulate propolis extracts in lipophilic polymercarriers using alternative propolis extract to polymer ratios - 1/4, 1/2 and 3/4 - by double emulsion solvent evaporation approach. The product yield was higher than the previously reported in the literature $(80.3 \pm 3.7 \%$ versus $68 \%)$.

The encapsulation efficiencies (considering the encapsulated antioxidants and phenolic compounds) were independent from the propolis extract to polymer ratio, for the studied lipophilic polymer-carriers. The results of this work provide promising approaches for the manufacture of propolis extracts-loaded microparticles for further incorporation in functional matrices.
\end{abstract}

(c) 2021 Institution of Chemical Engineers. Published by Elsevier B.V. All rights reserved.

\section{Introduction}

Propolis is a natural heterogeneous complex matrix that is produced by honeybees by mixing resin, beeswax and other bees' own substances (Catchpole et al., 2018; Daugsch et al., 2008; Vyssotski et al., 2018). Several types of propolis were described in the literature, being characterized by their chemical diversity, geographical origin, and botanical sources (Ankovaa et al., 2000; Bankova, 2005; Gómez-Caravaca

\footnotetext{
* Corresponding author.

E-mail address: lsantos@fe.up.pt (L. Santos). https://doi.org/10.1016/j.fbp.2021.03.019
}

0960-3085/@ 2021 Institution of Chemical Engineers. Published by Elsevier B.V. All rights reserved. et al., 2006; Zabaiou et al., 2017). Despite the variation, propolis from different geographical origins exhibit a similar global physicochemical composition, containing $50 \%$ of resin, $30 \%$ of wax, $10 \%$ of essential oils, $5 \%$ of pollen and $5 \%$ of other organic compounds (Cheng et al., 2013; GómezCaravaca et al., 2006; González-búrquez et al., 2018; Pellati et al., 2013; Zabaiou et al., 2017). The resin is roughly composed by polyphenols and related phenolic acids which, alongside with flavonoids, present proven bioactive activities like (i) the ability to inhibit specific enzymes, (ii) to stimulate specific hormones, (iii) to trigger the activity of some neurotransmissions and (iv) to scavenge radical species (Gómez-Caravaca et al., 2006). Red propolis, found mainly in the northeast 
Brazil, has a distinct profile of biologic compounds, possessing flavonoids that are not detected in other types of propolis, such as biochamine A, formononetine, liquiritigenin and medicarpine (Santos et al., 2020). Other studies have also emphasized the higher concentration of isoflavonoids, pterocarpanes, chalcones, preny-lated benzophenones, terpenes and tannins in the type of propolis (Freires et al., 2016).

The bioactive compounds of propolis have been reported to be responsible for its beneficial bioactivities, among which antibacterial, antifungal, antiviral, antioxidant, antiinflammatory, antiulcer, anticarcinogenic, cytostatic and photoinhibitior, being a promising matrix for food, pharmaceutical, and even for agricultural industries (Bodini et al., 2013; Carvalho et al., 2004; Ely and Moura, 2017; Luiza et al., 2011; Mahadik and Paradkar, 2015; Milane et al., 2015; Nazareno, 2009; Ordó et al., 2011; Oryan et al., 2018; Santos et al., 2020; Valenzuela-barra et al., 2015).

However, despite these outstanding health benefits, practical propolis' application is still limited due to the high relative hydrophilicity (Busch et al., 2017) and, particularly within the context of food industry, to its strong and unpleasant taste and aroma (Bodini et al., 2013; Busch et al., 2017; Nori et al., 2011). Also, propolis' phenolic compounds have unsaturated bonds in their structure which make them very sensitive to oxidizing environments as light, moisture, oxygen and heat (Ballesteros et al., 2017); therefore, these may not present long term stability which hampers propolis inclusion in functional matrices (Rocha-parra et al., 2016).

In this context, microencapsulation arises as an approach to protect propolis phenolic compounds and to preserve these during mid to long term incorporation in complex lipophilic matrices, like food products, while allowing their controlled release. Moreover, microencapsulation may aid to mask the unpleasant flavor and aroma of propolis (Paulo and Santos, 2018a,b, 2019, 2020a,b). Several methods have been used for the microencapsulation of bioactive compounds. Among them, water-in-oil-in-water $\left(\mathrm{w}_{1} / \mathrm{o} / \mathrm{w}_{2}\right)$ double emulsion solvent evaporation technique allows entrapping hydrophilic compounds protecting them from oxidizing and allowing a sustained release in lipophilic matrices ( $\mathrm{Wu}$ and Meininger, 2002). Another advantage is that this technique can be performed at room temperature - unlike other widely employed methodologies such as spray-drying and high pressure homogenization encapsulation techniques (Paulo and Santos, 2018a,b, 2019, 2020a,b).

Several biocompatible polymers may be used as coating materials for the encapsulation of high to relative water-soluble compounds; one of the most used is poly(D,Llactide-co-glycolide) (PLGA), a synthetic biodegradable and biocompatible copolymer of poly lactic acid (PLA) and polyglycolic acid (PGA) (Han et al., 2016). This copolymer has unique characteristics, being possible to control in a very sensitive way the physicochemical interactions between the copolymer and the incorporated compounds by monitoring the polymer molecular weight, the lactide to glycolide ratio, encapsulated material concentration, among other parameters (Han et al., 2016). Alternative water-insoluble biocompatible polymers have been studied, including the semi-synthetic polymer ethylcellulose (EC) and the synthetic polymer polycaprolactone (PCL) (Durán et al., 2007; Lecomte et al., 2003). Ethylcellulose has been extensively studied as a coating material of bioactive compounds for food industry. Moreover, studies have been also reported the effective protection of bioactive compounds using PCL (Avanço and Braschi, 2008; Paulo and Santos, 2018a,b, 2019, 2020a,b).

In this context, this study aimed to obtain propolis extracts-loaded microparticles using three different polymeric matrices (poly(D,L-lactide-co-glycolide), ethylcellulose and polycaprolactone) and three propolis extracts to polymers ratio $(1 / 4,1 / 2$ and $3 / 4)$, using the $\mathrm{w}_{1} / \mathrm{o} / \mathrm{w}_{2}$ double emulsion solvent evaporation technique. This procedure was expected to protect the biological compounds contained in propolis samples while bypassing the acceptance problems due to its unpleasant sensory characteristics. Microparticles were then evaluated regarding the product yield, the external morphology, and the particle size distribution. The quantitative evaluation of the encapsulation efficiency was performed considering the total amount of antioxidants, and phenolic compounds encapsulated, while the qualitative evaluation of the inclusion of bioactive compounds was assessed using the Fourier transformed infrared spectroscopy (FTIR).

To the authors best knowledge this is the first time that a comprehensive physicochemical characterization of propolisloaded microparticles is performed. Moreover, this is the first time that three polymer-carriers and three loadings (propolis to polymer ratio) are studied simultaneously. The results of this work provide promising insights for the obtainment of propolis extracts-loaded microparticles for further incorporation in functional matrices (e.g., foods).

\section{Materials and methods}

\subsection{Chemicals, reagents, and samples}

\subsubsection{Propolis sample}

Red propolis was collected in April 2016 by scraping the walls and frames of Apis mellifera beehives. The material (4 samples, $250 \mathrm{~g}$ each) was harvested in the private land from different producers from Canavieiras city, Bahia, in the Northeast region of the country, with owners' permission. Upon receipt, each sample was inspected in order to find rests of bees, wood, plant, among others. The major visible impurities were removed from the samples; none of the samples had signs of visible contamination, fermentation, or spoilage. The samples harvested by different producers were then mixed into a pool in order to obtain a composite sample and frozen at $-20^{\circ} \mathrm{C}$ until further use.

\subsubsection{Chemicals}

The polymers, ethylcellulose (Ref. 433837-250G, viscosity of 46 cP, CAS 3004-57-3), poly(D,L-lactide-co-glycolide) (Resomer ${ }^{\circledR}$ RG 504, Ref: $739944-5 G$, viscosity of $0.45-0.6 \mathrm{dL} / \mathrm{g}$, CAS 26780 50-7, lactide/glycolide 50/50) and polycaprolactone (Ref. 440744-250G, CAS 24980-41-4), used as coating materials for the encapsulation of propolis extract were purchased from Sigma Aldrich Chemical (Merck KGaA, Darmstadt, Germany). Moreover, polyvinyl alcohol used as the surfactant of the water-in-oil-in-water double emulsion (Ref: P8136250G, 87-90\% hydrolyzed, the average molecular weight of 30,000-70,000, CAS 9002-89-5) was also purchased from Sigma Aldrich Chemical (Merck KGaA, Darmstadt, Germany). The solvent chosen for the formulation of all oily phases was dichloromethane, and it was obtained from VWR International (Fontenay-sous-Bois, France).

The total antioxidant activity of the obtained microparticles was evaluated using a total antioxidant capacity assay kit 
(Ref. MAK187) purchased to Sigma-Aldrich Chemical (Merck $\mathrm{KGaA}$, Darmstadt, Germany), using the colorimetric method and trolox as standard according to manufacturer instructions.

The total amount of phenolic compounds encapsulated in the polymeric matrices was evaluated using the Folin-Ciocalteu reagent (Ref. 47641-500ML-F, $2 \mathrm{M}$ with respect to acid, density of $1.27 \mathrm{~g} / \mathrm{mL}$ at $20^{\circ} \mathrm{C}$ ) and anhydrous sodium carbonate (Ref: 27767.295, $\mathrm{Na}_{2} \mathrm{CO}_{3}$, assay 99.5 to $100.5 \%$ Ph.Eur., molecular weight of $105.99 \mathrm{~g} / \mathrm{mol}$, CAS 49719-8). The Folin-Ciocalteu reagent was obtained from to Sigma-Aldrich Chemical (Merck KGaA, Darmstadt, Germany) and the anhydrous sodium carbonate was purchased from VWR International (Fontenay-sous-Bois, France).

For the dissolution of the obtained extracted was used a mixture of ethanol/ultrapure water $(70 / 30, v / v)$. Ethanol ( $\geq 99.9 \%$, by GC, LiChrosolv ${ }^{\circledR}$, liquid chromatography grade) was obtained from VWR International (Fontenay-sous-Bois, France).

Methanol $\left(\geq 99.9 \%\right.$, EMSURE $^{\circledR}$ ACS, ISO, Reag. Ph. Eur. for analysis, Supelco ${ }^{\circledR}$ ) used for extraction was supplied by VWR International (Fontenay-sous-Bois, France).

The water used in this work was de-ionized and doubledistilled using a Millipore ${ }^{\mathrm{TM}}$ water purification system (Massachusetts, USA) having $18.2 \Omega$ electrical resistivity.

All the reagents were either chromatographic or analytical grade and used as received.

\subsection{Methods}

\subsubsection{Analytical methods validation}

The ultraviolet-visible (UV-vis) spectrophotometer method was employed on a UV-vis spectrophotometer V-530 (Jasco, OK, USA) at $760 \mathrm{~nm}$ using quartz cells of $10 \mathrm{~mm}$ of a light path for the quantification of the total phenolic content of propolis extracts-loaded microparticles. The SPECTA MANAGER software was used for the absorbance measurements.

The quantification of the antioxidant capacity of propolis extracts-loaded microparticles, the ultraviolet-visible (UV-vis) spectrophotometer method was also employed. In this case, the absorbance was read at $570 \mathrm{~nm}$ in a microtiter plate reader (Synergy HT, Biotek, USA). The OPUS software was used for absorbance measurements.

The experiments were conducted similarly as described by Paulo and Santos (2018a,b, 2019).

\subsubsection{Extraction of propolis samples}

The main biologically active compounds present in propolis are scarcely soluble in water, oil and other solvents commonly used in the industry. The most popular technique used to obtain propolis extracts is ethanol extraction. However, although this is a simple and effective method it has disadvantages such as a strong residual flavor and limitations in the application in the food, cosmetics and pharmaceutical industry (Kubiliene et al., 2015). According to the literature, propolis methanolic extracts possess higher total phenolic and flavonoid contents and biological activities in comparison with those of extracts obtained using other solvents (Karapetsas et al., 2019). In this study, methanolic extracts were obtained as described by Silva et al. (2012), with some modifications. The propolis samples were divided into small pieces, weighed and diluted in methanol in a ratio of 1:5 and stirred for $24 \mathrm{~h}$. The mixtures were filtered (Whatman \# 4 filter paper), and the residue was re-extracted following the same procedure. The methanolic extracts were then placed under refrigeration and after $12 \mathrm{~h}$ filtrated again. Subsequently, the solvent was evaporated on a rotary evaporator (Rotavapor Buchi RE 111 with a Buchi 461 water bath). The residue was stored in the greenhouse and weighted to constant weight.

\subsubsection{Preparation of propolis extracts-loaded}

microparticles

Propolis extracts-loaded microparticles were formulated by water-in-oil-in-water $\left(\mathrm{w}_{1} / \mathrm{o} / \mathrm{w}_{2}\right)$ double emulsion solvent evaporation technique as described by Paulo and Santos (2018a,b).

In the present study, three different polymers namely poly(D,L-lactide-co-glycolide) (PLGA), ethyl cellulose (EC) and polycaprolactone (PCL) (with different hydrophilic properties; hydrophilicity: PLGA > EC > PCL) were considered. Moreover, different propolis extract to polymer ratios (PPR), namely $1 / 4$, $1 / 2$, and $3 / 4$, were evaluated for each polymer.

The obtained extract of the raw propolis was dissolved in a solvent mixture of 70/30 ethanol/water. Generally, it is desired a complete immiscibility among the internal aqueous phase and the organic phase, however, due to the limited solubility of the propolis extract in water, a solvent ethanol/water mixture of 70/30 was considered in this study.

For this purpose, the extract was reconstituted in $3 \mathrm{~mL}$ of the solvent mixture ethanol/water. The extract solution was diluted according to the PPR. The obtained diluted extract solutions constituted the internal aqueous phases (w1). In this context, for each formulation, a mass of $100 \mathrm{mg}$ of ethylcellulose was dissolved in $10 \mathrm{~mL}$ of dichloromethane to form the polymer solution used as the oily phase (o) to achieve a polymer concentration of $10 \mathrm{~g} / \mathrm{L}$. The obtained polymer solution was sonicated for $15 \mathrm{~min}$ in an ultrasonic bath. Then, a volume of $1 \mathrm{~mL}$ of $\mathrm{w} 1$ was added to the polymer solution. To obtain a stable w1/o stable primary emulsion, the mixture was vortexed for 5 minutes. Afterward, the w1/o solution was poured into $100 \mathrm{~g}$ of PVA solution (concentration of $1 \%, w / w)$ and emulsified using a high-performance homogenizer (T18 digital ULTRA-TURRAX ${ }^{\circledR}$, IKA, Staufen im Breisgau, Baden-Württemberg, Germany) at $5000 \mathrm{rpm}$ for $5 \mathrm{~min}$. The final mixture (w1/o/w2 double emulsion) was continuously mixed in a stirring plate at $700 \mathrm{rpm}$ for $3 \mathrm{~h}$ in the fume hood at room temperature $\left(20 \pm 2^{\circ} \mathrm{C}\right)$ to promote the solvent evaporation and, therefore, the hardening of microparticles. Microparticles were then recovered by filtration using a $0.2 \mu \mathrm{m}$ quantitative paper filter and washed with $500 \mathrm{~mL}$ of distilled water. The recovered microparticles were frozen for $24 \mathrm{~h}$ at $-22^{\circ} \mathrm{C}$ and freeze-dried for $72 \mathrm{~h}$.

Therefore, in this study, the effectiveness of the encapsulation of propolis extract was evaluated using three polymers, each at three different propolis extract to polymer ratio levels as presented in Table 1 . All the experiments were performed in triplicate in a total formulation runs of 27 experiments. Further considerations are presented in Table 1. A thermogravimetric analysis (TGA) was performed in order to evaluate the residual presence of solvents on the formulated microparticles (data not shown). The powders did not present residual solvent as no weight loss was verified in the TGA thermograms of the powders obtained at the temperatures corresponding to the boiling point of the solvents - dichloromethane and ethanol. 


\section{Table 1 - Experimental conditions of the study.}

\begin{tabular}{lllll} 
Entry & Polymer & PPR (w/w) & $\begin{array}{l}\text { Polymer conc. } \\
(\mathrm{g} / \mathrm{L})^{\mathrm{a}}\end{array}$ & $\begin{array}{l}\text { PVA conc. } \\
\mathrm{w} / \mathrm{w})^{\mathrm{b}}\end{array}$ \\
\hline 1 & PLGA & $1 / 4$ & 10 & 1 \\
2 & & $1 / 2$ & & \\
3 & & $3 / 4$ & & \\
4 & EC & $1 / 4$ & 10 & 1 \\
5 & & $1 / 2$ & & \\
6 & & $3 / 4$ & & 1 \\
7 & PCL & $1 / 4$ & 10 & \\
8 & & $1 / 2$ & & \\
9 & & $3 / 4$ & & \\
\hline
\end{tabular}

${ }^{a}$ Polymer concentration expressed in grams (g) of polymer in the solvent - dichloromethane $(\mathrm{mL})$

b PVA concentration is related to external water phase.

Conc. - concentration; EC - ethyl cellulose; PCL - polycaprolactone; PLGA - poly-(D,L-lactide-co-glycolide); PPR - propolis to polymer ratio; PROP - propolis; PVA - polyvinyl alcohol.

\subsection{Physicochemical characterization of the propolis extracts-loaded microparticles}

\subsubsection{Assessment of the product yield}

The product yield was assessed as described by Paulo and Santos (2018a,b). The product yield was evaluated according to Eq. (1) as follow:

$P Y=\frac{m_{M}}{m_{A}}=\frac{m_{M}}{m_{P}+m_{I}}=\frac{m_{M}}{m_{P}+\alpha m_{P}}$

where $m_{M}$ represents the mass of microparticles recovered after the freeze-drying process and $m_{A}$ the sum of the mass of polymer $\left(m_{P}\right)$ (PLGA, EC or PCL) added to the formulation and $m_{\mathrm{I}}$ the mass of the propolis sample initially added to the respective formulation, corresponding to $1 / 4,1 / 2$ or $3 / 4(\alpha)$ of the polymer mass $\left(m_{P}\right)$.

\subsubsection{Assessment of propolis extracts-loaded} microparticles morphology

The external morphology of propolis extracts-loaded microparticles was evaluated according to Paulo and Santos (2018a). For that purpose, propolis extracts-loaded microparticles morphological and polydispersity characteristics were assessed using a PHENOM XL scanning electron microscope (Eindhoven, The Netherlands) at an accelerating voltage of $10 \mathrm{kV}$. Freeze-dried microparticles were placed on an aluminum stub with a carbon double-sided adhesive tape.

The samples were sputter-coated with gold for $20 \mathrm{~s}$ using a vacuum-sputtering coater (Leica, EM SCD 500, Wetzlar, Germany).

\subsubsection{Assessment of the propolis extracts-loaded particle size distribution}

The laser granulometry technique was employed to evaluate the particle size distribution of propolis extracts-loaded microparticles. A Coulter Counter-LS 230 Particle Size Analyzer (Miami, FL, USA) equipment was used. The differential volume distribution was considered, recording the particle size distribution. Particle size distribution and polydispersity were evaluated for each formulation in triplicate. Each sample (about $100 \mathrm{mg}$ ) was dispersed in $25 \mathrm{~mL}$ of ultrapure water before analysis. Average values were considered in data analysis. The polydispersity of particle size distributions was analyzed considering the polydispersity index (PDI) as demonstrated in Eq. (2):

$P D I=\frac{D_{v, 90}-D_{v, 10}}{D_{v, 50}}$

where $D_{v, 90}, D_{v, 10}$ and $D_{v, 50}$ represent respectively, the maximum particle diameter below which $90 \%$ of the sample volume exists, the maximum particle diameter below which $10 \%$ of the sample volume exists and the maximum particle diameter below which $50 \%$ of the sample volume exists.

\subsection{Quantitative assessment of antioxidant activity encapsulation efficiency of propolis extracts-loaded microparticles}

\subsubsection{Determination of the total antioxidant}

encapsulation efficiency

The total amount of antioxidants encapsulated using the $\mathrm{w}_{1} / \mathrm{o} / \mathrm{w}_{2}$ double emulsion solvent evaporation technique was evaluated using the total non-enzymatic antioxidant capacity (TAC) kit (Ref. MAK187, Sigma-Aldrich Chemical, St. Louis, MO, USA).

After the hardening of microparticles $(3 \mathrm{~h}$ after the placement of the $\mathrm{w}_{1} / \mathrm{o} / \mathrm{w}_{2}$ double emulsion in the fume hood under constant magnetic stirring at $700 \mathrm{rpm}$ ), a sample of microparticles was taken and submitted a low intensity centrifugation process at $4000 \mathrm{rpm}(2670 \mathrm{G})$ for $30 \mathrm{~min}$. Afterward, the supernatant was recovered to further determination of the total amount of non-encapsulated antioxidants (recovered sample from the supernatant $-R_{S}$ ). The pellet was reconstituted in a solvent mixture in which the compounds present in propolis extract are soluble (ethanol/water, 70/30). The reconstructed pellet was further submitted to a centrifugation process in the same conditions above reported in order to allow the transference of antioxidants from the surface of microparticles to the solvent mixture (recovery sample from the reconstitution of the pellet $-R_{P}$ ).

The total antioxidant encapsulation efficiency (TAEE) was evaluated, as presented in Eq. (3):

$\mathrm{TAEE}=\frac{m A_{P R O P}}{m A_{I}}=\frac{m A_{I}-m A_{O}}{m A_{I}}=\frac{m A_{I}-m A_{P T}-m A_{S}}{m A_{I}}$

where $m A_{P R O P}$ corresponds to the mass of antioxidants present in propolis encapsulated. The $m A_{P R O P}$ can be defined as the difference between the mass of antioxidants in propolis initially added for the encapsulation process $\left(m A_{I}\right)$ and the mass of bioactive antioxidant compounds no encapsulated $\left(m A_{0}\right)$. The $m A_{0}$ corresponds to the sum of the mass of antioxidant bioactive compounds found in the pellet $\left(m A_{P T}\right)$ and the amount of these bioactives found in the supernatant $\left(m A_{S}\right)$.

The protocol previously described by Paulo and Santos (2018a,b) was adapted to obtain the required data for TAEE calculation. For that, $100 \mu \mathrm{L}$ of filtrated samples (propolis extract in the case of $m A_{I}$, the supernatant and the pellet after encapsulation, corresponding to $m_{S}$ and $m_{P T}$ respectively) were transferred to a 96 well plate. Then after, $100 \mu \mathrm{L}$ of $\mathrm{Cu}^{2+}$ working solution $\left(\mathrm{Cu}^{2+}\right.$ concentration of $\left.2 \%, \mathrm{v} / \mathrm{v}\right)$ was added to all sample wells. The content of each well was mixed by pipetting and incubated for $90 \mathrm{~min}$, in the dark and at room temperature. Afterward, the absorbance was read at $570 \mathrm{~nm}$ in a microtiter plate reader (Synergy HT, Biotek, USA).

The mass values $\left(m A_{0}, m A_{S}\right.$ and $\left.m A_{P T}\right)$ were obtained after considering the antioxidant concentration at each well 
(ACW) and the analyzed volume. The ACW was evaluated according to Eq. (4) as follows:

AWC $(n m o l / \mu L$ or $m M$ Trolox Equivalent $)=\frac{S_{a}}{S_{v}}$

where $S_{a}$ corresponds to the Trolox equivalent of the analyzed sample in the well (nmole) from the standard calibration curve and $S_{v}$ is the sample volume $(\mu \mathrm{L})$ added to the well.

Previously, standard Trolox calibration curves were obtained adding $0,4,8,12,16$ and $20 \mu \mathrm{L}$ of the $1 \mathrm{mM}$ of the Trolox Standard Solution into a 96 well plate. Ultra-pure water or ethanol/ultrapure water were added to each well to bring to volume to $100 \mu \mathrm{L}$. The $0,4,8,12,16$, and $20 \mathrm{nmol} /$ well standards were generated. All the measurements were performed in triplicate.

\subsubsection{Determination of the total phenolic encapsulation efficiency}

The total phenolic encapsulation efficiency (TPEE) was evaluated similarly to the TAEE. For that, aliquots of the same samples obtained after the hardening and centrifuging of the microparticles $\left(R_{S}\right)$. The centrifuged reconstructed pellet after the second centrifugation were considered in the following study.

To each recovered aliquot, the Folin-Ciocalteu procedure was applied as firstly described by Singleton and Rudolf Lamuela-Raventós (1999) with slight modifications. A 10-fold (1:10) scale down was considered to a final volume of $10 \mathrm{~mL}$. For that, to a $0.1 \mathrm{~mL}$ of standard solution (gallic acid in ultrapure water or gallic acid in a mixture of 70/30 ethanol/ultrapure water) or sample was added $6.0 \mathrm{~mL}$ of ultrapure water and $0.5 \mathrm{~mL}$ of the Folin-Ciocalteu reagent (Ref. 47641-500ML-F, $2 \mathrm{M}$ with respect to acid, density of $1.27 \mathrm{~g} / \mathrm{mL}$ at $20^{\circ} \mathrm{C}$ ), the mixture was vortexed for $1 \mathrm{~min}$ and incubated for $5 \mathrm{~min}$ in the dark, at room temperature. Afterward, $1.5 \mathrm{~mL}$ of anhydrous sodium carbonate $(20 \%, w / v)$ was added. Then, ultrapure water was added to complete the volume of $10 \mathrm{~mL}$. The final solution was again vortexed for $1 \mathrm{~min}$ and incubated, being also light protected at room temperature for $2 \mathrm{~h}$.

Afterwards, the sample/standard was analyzed using the UV-vis spectrophotometer V-530 (Jasco, OK, USA) at $760 \mathrm{~nm}$ using quartz cells of $10 \mathrm{~mm}$ of a light path. A blank solution was considered following the reported procedure but replacing the volume of the sample/standard $(0.1 \mathrm{~mL})$ by the same volume of ultrapure water. The phenolic encapsulation efficiency was calculated according to Eq. (5) as described:

$\mathrm{TPEE}=\frac{m P_{P R O P}}{m P_{I}}=\frac{m P_{I}-m P_{O}}{m P_{I}}=\frac{m P_{I}-m P_{P T}-m P_{S}}{m P_{I}}$

where $m P_{P R O P}$ is the mass of phenolic compounds encapsulated and $m P_{I}$, the mass of phenolic compounds used for the encapsulation, this is the phenolic content of the extract, determined by means of the Folin-Ciocalteu total phenolic determination. The $\mathrm{MP}_{\mathrm{PROP}}$ corresponds to the mass different of the total phenolic content between the sample initialy added for the encapsulation $m P_{I}$ and the total phenolic mass no encapsulated $\left(m P_{0}\right)$. The $\mathrm{WP}_{0}$ corresponds to the sum of the mass of total phenolic compounds present in the $R_{S}$ and $\mathrm{R}_{\mathrm{P}}$ samples: $m P_{S}$ and $m P_{P T}$, respectively.

The standard gallic acid was chosen for the obtainment of standard calibration curves using the Folin-Ciocalteu method. Even though other standards can be used for the drawn of calibration curves, gallic acid is the most widely used standard for this procedure. Moreover, it is not an expensive standard, it is soluble in water and is easily recrystallized from water and dried, being stable in the dry form (Singleton and Rudolf Lamuela-Raventós, 1999).

Two alternative calibration curves were obtained for the determination of phenolic compounds encapsulation efficiency. A calibration curve of gallic acid in ultrapure water and a calibration curve of gallic acid in a solvent mixture of ethanol/water (70/30) were obtained for the determination of the total phenolic content in $R_{S}$ samples and in $R_{P}$ samples, respectively.

\subsection{Qualitative assessment of bioactive compounds and antioxidants encapsulation efficiency of propolis extracts-loaded microparticles}

\subsubsection{Fourier transform infrared spectroscopy}

The qualitative assessment of bioactive compounds encapsulation efficiency of propolis extracts-loaded microparticles was evaluated by Fourier transform infrared spectroscopy (FTIR) method. The infrared spectra were recorded using a VERTEX 70 FTIR spectrometer (BRUKER) in transmittance mode with a high sensitivity DLaTGS detector at room temperature. Samples were measured in attenuated total reflectance (ATR) mode, with an A225/Q PLATINUM ATR Diamond crystal with single reflection accessory. The spectra were recorded from 4000 to $500 \mathrm{~cm}^{-1}$ with a resolution of $4 \mathrm{~cm}^{-1}$.

The presence of bioactive compounds with different functional groups of the encapsulating material used (PLGA, EC or PCL) indicate the possibility of surface adsorption of these compounds, demonstrating that there were not incorporated in the polymeric matrix. Moreover, the comparison of FTIR spectra of unloaded microparticles and propolis sample (extract) allows supporting the theses taken by the study of FTIR spectra of propolis extracts-loaded microparticles.

\subsection{Statistical analysis}

All the experiments were performed in triplicate. Results are present as mean \pm standard deviation. The analysis of variance (ANOVA) was used to determine significant differences $(p<0.05)$.

\section{Results and discussion}

\subsection{Analytical methods validation}

The methods developed for the quantification of the total amount of antioxidants and phenolic compounds encapsulated were validated regarding the linearity ranges, coefficients of determinations, limits of detection (LODs) and quantification (LOQs). The results regarding the analytical methods validation are presented in Table 2.

For each detection medium, a calibration curve was drawn. In the case of the quantification of antioxidants, a linear range was observed, and the calibration curves were constructed in the range of $0-20 \mathrm{nmol} / \mu \mathrm{L}$. In the case of the detection of phenolic compounds, the calibration curves were drawn using gallic acid as a standard, and a linear range was observed between 0.250 and $30 \mathrm{mg} / \mathrm{L}$.

The LOD and LOQ values for the calibration curves were acceptably low, demonstrating the possibility of their appli- 
Table 2 - Results from the regression analysis for the total amount of antioxidants and phenolic compounds encapsulated using different polymeric carriers.

\begin{tabular}{|c|c|c|c|c|c|c|c|c|}
\hline Wavelength & $\begin{array}{l}\text { Compounds } \\
\text { detected }\end{array}$ & $\begin{array}{l}\text { Standard } \\
\text { (DF) }\end{array}$ & Regression equation ${ }^{\mathrm{a}, \mathrm{b}}$ & $R$ & $\mathrm{~S}_{\mathrm{a}} / \mathrm{a}(\%)$ & Conc. range $^{c}$ & $\mathrm{LOD}^{\mathrm{c}}$ & $\mathrm{LOQ}^{\mathrm{C}}$ \\
\hline \multirow[t]{2}{*}{570} & \multirow[t]{2}{*}{ Antioxidants } & $\begin{array}{l}\text { Trolox } \\
\text { (UPW) }\end{array}$ & $\begin{array}{l}\text { Abs }=(0.028 \pm 0.003) C+ \\
(0.014 \pm 0.040)\end{array}$ & 0.997 & 3.456 & $1.8-20$ & 1.375 & 2.979 \\
\hline & & $\begin{array}{l}\text { Trolox } \\
\text { (EtOH/UPW } \\
\text { 70/30, v/v) }\end{array}$ & $\begin{array}{l}\text { Abs }=(0.037 \pm 0.003) C+ \\
(0.008 \pm 0.036)\end{array}$ & 0.999 & 2.246 & $2.1-20$ & 0.894 & 2.979 \\
\hline \multirow[t]{2}{*}{760} & \multirow[t]{2}{*}{$\begin{array}{l}\text { Phenolic } \\
\text { com- } \\
\text { pounds }\end{array}$} & $\begin{array}{l}\text { GAL } \\
(\mathrm{UPW})\end{array}$ & $\begin{array}{l}\text { Abs }=(0.108 \pm 0.003) C+ \\
(0.009 \pm 0.048)\end{array}$ & 0.998 & 1.430 & $0.650-30$ & 0.595 & 1.983 \\
\hline & & $\begin{array}{l}\text { GAL } \\
\text { (EtOH/UPW } \\
70 / 30, v / v)\end{array}$ & $\begin{array}{l}\text { Abs }=(0.065 \pm 0.003) C+ \\
(0.009 \pm 0.025)\end{array}$ & 0.996 & 2.223 & $0.650-30$ & 0.502 & 1.673 \\
\hline \multicolumn{9}{|c|}{$\begin{array}{l}\text { abs is the absorbance (UV-vis spectrophotometer method), and C is the Trolox Equivalents Concentration }(\mathrm{nmol} / \mu \mathrm{L}) \text { in the case of the detection } \\
\text { of antioxidants and C is the Gallic Equivalents Concentration }(\mathrm{mg} / \mathrm{L}) \text { in the case of the detection of phenolic compounds. } \\
\text { b Calibration curve based on three absorbances read. } \\
\text { c Presented in Trolox Equivalents Concentration }(\mathrm{nmol} / \mu \mathrm{L}) \text { in the case of the detection of antioxidants and Gallic Equivalents Concentration } \\
\text { (mg/L) in the case of the detection of phenolic compounds. } \\
\text { Abs - absorbance; Conc. - concentration; DT - detection fluid; EtOH - ethanol; Gal - gallic acid; LOD - limit of detection; LOQ - limit of } \\
\text { quantification; R - correlation coefficient; } \mathrm{S}_{\mathrm{a}} / \mathrm{a} \text { - relative standard error of the slope; UPW - ultrapure water. }\end{array}$} \\
\hline
\end{tabular}

cation for the quantification of antioxidant compounds and phenolic compounds present in propolis samples.

\subsection{Physicochemical characterization of propolis extracts-loaded microparticles}

\subsubsection{Assessment of the product yield}

Propolis extracts-loaded microparticles were produced in a two-step emulsification process. Firstly, the $\mathrm{w}_{1} / \mathrm{o}$ primary emulsion was obtained, and then the primary emulsion was re-emulsified in a dispersive phase $\left(\mathrm{w}_{2}\right)$. After the hardening of microparticles, they were recovered and freeze-dried. The obtainment of a dried particulated system is crucial for microparticles inclusion in functional matrices (e.g., foods) as it may improve the shelf life of foodstuff due to the low water content and the continuous release of antioxidants that may prevent the degradation of other vital molecules.

The mean production yields of propolis extracts-loaded ethylcellulose microparticles with a propolis extract to polymer ratio of $1 / 4,1 / 2$ and $3 / 4$ were $80.3 \pm 3.7 \%, 77.2 \pm 1.6 \%$ and $65.9 \pm 2.8 \%$, respectively.

Regarding the product yield results of propolis extractsloaded poly(D,L-lactide-co-glycolide) microparticles with a propolis extract to polymer ratio of $1 / 4,1 / 2$ and $3 / 4$ were $76.2 \pm 4.4 \%, 77.8 \pm 8.1 \%$ and $67.1 \pm 4.1 \%$, respectively.

In the case of propolis extracts-loaded polycaprolactone microparticles formulated with a propolis extract to polymer ratio of $1 / 4,1 / 2$ and $3 / 4$, the mean production yields were $70.7 \pm 11.1 \%, 74.4 \pm 5.2 \%$ and $72.8 \pm 10.2 \%$, respectively.

The results of this study demonstrate that in the case of propolis extracts-loaded ethylcellulose microparticles, the propolis extract to polymer ratio affected the production yield as was observed a statistically significant difference between the mean production yield among the propolis extract to polymer ratio tested $(p=0.005<0.050$, considering a significance level of $95 \%$ ).

The production yield of propolis extracts-loaded microparticles obtained using poly(D,L-lactide-co-glycolide) or using polycaprolactone seemed not to be affected by the propolis extract to polymer ratio as there were no observed differences between the experimental groups (grouped by the propolis extract to polymer ratio) as the $p$-values obtained were 0.222 and 0.924 for poly(D,L-lactide-co-glycolide) and polycaprolactone microparticles.

The product yield was only affected by the propolis extract to polymer ratio - decrease with the increase of the propolis to polymer ratio - in the case of ethylcellulose. In the case of PCL and PLGA, the influence of the PPR on yield of microparticles was not observed. It can be hypothesized that propolis extractethylcellulose can establish interactions, namely by hydrogen bonds affecting the yield of production. Probably, increasing the amount of propolis extract, the available sites to establish hydrogen bonds with EC are all filled, and therefore, molecules from propolis extract are released, decreasing, thus, the yield of production. In the case of PCL and PLGA, the lack of interaction sites with propolis is the main reason why the product yield of loaded PCL and PLGA microparticles is not affected by the PPR.

The researchers Jansen-alves et al. (2019) encapsulated propolis with pea protein using the spray-drying technique. During their studies regarding the characterization and evaluation of the antimicrobial and antioxidant potential of the microparticles formulated, they obtained production yield values ranging between $18.82 \%$ and $54.00 \%$. The highest production yield of propolis extracts-loaded microparticles $(54.00 \%)$ was obtained when they obtained the formulations with a wall material concentration of $6 \%$ and propolis extract of $5 \%$. They also found a direct correlation between the production yield and the concentration of propolis extract for the formulations obtained. They also addressed that the low production yield values obtained are in accordance with the results they found on literature regarding the use of isolated proteins as wall materials combined with the spray-drying technique. The adherence of microparticles in the atomizer chamber in the spray-dryer increased with the increase of the protein concentration, which led to a decrease of the production yield with the increase of protein concentration. The authors concluded that the use of the spray-drying approach was not suitable for the incorporation of propolis extracts using isolated proteins as wall materials.

An alcohol-free propolis powder was obtained by Busch et al. (2017) using different maltodextrin matrices (with or 
without added gums). They obtained a production yield of $60 \%$ when they used as wall materials maltodextrin with vinal gum and $68 \%$ in the cases of propolis encapsulated into maltodextrin without added gum and maltodextrin mixed with Arabic gum. The authors reported that the obtained values for the production yield are within the typical range of values that are obtained in the encapsulation of other bioactive compounds/extracts using the spray-drying technique. Moreover, the authors, reported that the lowest production yield value was obtained when it was used maltodextrin with vinal gum as many technical problems were observed due to high viscosity of the vinal gum solution during two critical steps of the process (i) filtration of the polymeric solution/wall material solution and (ii) the drying of particles in the spray-drying (Busch et al., 2015, 2017).

The authors Bruschi et al. (2003), obtained propolis extracts-loaded gelatine microparticles using the spray-drying technique. They found a product yield ranging between $28 \%$ and $51 \%$. The maximum product yield was obtained using gelatine to propolis extract ratio of $6 / 1(\mathrm{w} / \mathrm{w})$ and a mannitol concentration of $20 \%$. The operational conditions of the spray-dryer were $160{ }^{\circ} \mathrm{C}, 6 \%, 80 \%$ and $3 \%$ for the inlet temperature, feed rate, aspiration, and pressure, respectively. Once again, the obtained values for the production yield are within the typical range values that are commonly obtained during the encapsulation by the spray-drying technique.

A pressurized carbon dioxide anti-solvent co-precipitation process was applied by Yang et al. (2014) for the encapsulation of propolis extracts with polyethylene glycol polymer. The preliminary results pointed out that an increase in propolis extract to polymer ratio led to a decrease in the product yield. The trend observed by Yang et al. (2014) was similar to the one observed in this study - using ethyl cellulose as the polymer carrier an increase of the propolis extract to polymer ratio lead to a decrease in the production yield (Fig. 1B)

The results of this study are significative as was obtained production yield values higher than the ones found in the literature. Comparing to the case of propolis extracts-loaded microparticles obtained by spray-drying, it can be stated that due to non-feasibility of the application of the spray-drying technique, mainly due to the (i) high viscosities of polymer solutions increase the number of particles retained in the drying chamber and (ii) typical low-values for the production yield are found using this approach, the $\mathrm{w}_{1} / \mathrm{o} / \mathrm{w}_{2}$ double emulsion solvent evaporation technique may be an alternative approach to overcome the existing drawbacks.

\subsubsection{Assessment of the morphology}

The microphotographs of propolis extracts-loaded microparticles are present in Table 3, at two different amplification levels (5000 times and 10,000 times; beam intensity of $10.00 \mathrm{kV}$ ).

It can generally state that for all the polymer-carriers (ethylcellulose poly(D,L-lactide-co-glycolide) and polycaprolactone), the obtained exhibited practically no pores and round shapes: spherical in the case of PLC-loaded microparticles and PLGA-loaded microparticles and bowl shape in the case of ECloaded microparticles. Nevertheless, the surface smoothness, polydispersity, and particle sizes depended on the polymer carrier and the PPR. However, it was no observed a typical morphological trend associated with the PPR values. Therefore, it can be concluded that microparticles morphological appearance was dependent on the type of the polymer carrier and the propolis extract to polymer ratio.
The almost total absence of dents - a morphological characteristic of microparticles when they present small concavities - indicate the slow and appropriate time for the solvent evaporation.

Moreover, the absence of pores, fissures, interruptions, and cracks - in the majority of microphotographs - guarantee the efficient retention and protection of propolis extract.

These morphological characteristics are typical among microparticles produced by double emulsion solvent evaporation technique using the high-performance liquid homogenizer for emulsification. In previous studies, were also verified these typical morphologic characteristics when the $\mathrm{w}_{1} / \mathrm{o} / \mathrm{w}_{2}$ double emulsion solvent evaporation technique was applied using a liquid homogenizer for the emulsification steps (Paulo and Santos, 2018a,b).

The morphological characteristics of propolis extractsloaded microparticles produced in alternative formulation and process conditions as well as intended for different final applications have been studied by many authors as follows: Bruschi et al. (2003), Busch et al. (2017), Da Silva et al. (2013), Dangui et al. (2017), Dota et al. (2011), Gomes et al. (2014), Jansen-alves et al. (2018, 2019), Jansen-alves and Zambiazi (2019), Onbas et al. (2016), Silva et al. (2011), Yang et al. (2014) and Zhang et al. (2018).

Microphotographs of propolis extracts-loaded microparticles obtained by spray-drying were presented by Silva et al. (2011) when they were studying the physicochemical properties, antioxidant activity, and stability of spray-dried propolis extracts-loaded microparticles. Even though the authors did not discuss the morphological characteristics of the obtained microparticles, it can be drawn that the obtained spraydried propolis extracts-loaded microparticles presented an amorphous morphology, not being possible to picture the differentiation of spheres. In the present study, even though microparticles present a high degree of agglomeration, it can be stated that against to the obtained results by Silva et al. (2011), there was observed an improvement of the morphological characteristics of propolis extracts-loaded microparticles which may be the explanatory reason for the improvement of the efficient incorporation of propolis extracts into microparticulated polymer-based systems.

A study regarding the encapsulation of a propolis extract into ethylcellulose microparticles using an emulsification technique with solvent evaporation was performed by Avanço and Bruschi (2008). According to the authors, the obtained microparticles were spherical and presented uniform morphological outer characteristics. Moreover, the authors recognized the tendency of microparticles to form clusters. The results presented in this study are in agreement with those presented by Avanço and Bruschi (2008) - microparticles tend to be assembled in clusters.

The authors Durán et al. (2007) produced spherical and smooth microparticles using polycaprolactone as polymer and reported that polyvinyl alcohol could help obtain the spherical form and a low distribution size profile. However, further discussion regarding the morphological characteristics of microparticles was not presented by the authors. Comparing the microphotographs of microparticles obtained by Durán et al. (2007) and the ones obtained in this study using polycaprolactone as the encapsulating material (entries 7-9 from Table 3), the outer morphology was similar - however, insights regarding the distribution of microparticles in clusters is not illustrated in the microphotograph presented in the study of Durán et al. (2007). 
Table 3 - Scanning light microscopy micrographs of the propolis extracts-loaded microparticles.

No.

Pol.

PLGA

$1: 4$

$1: 2$
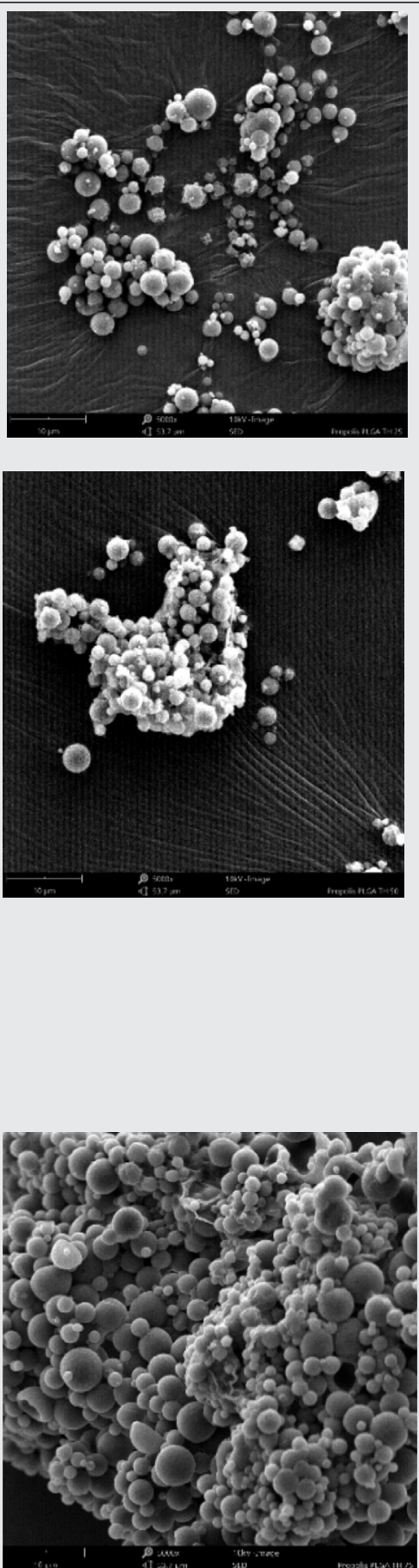

Amp. 10,000 times
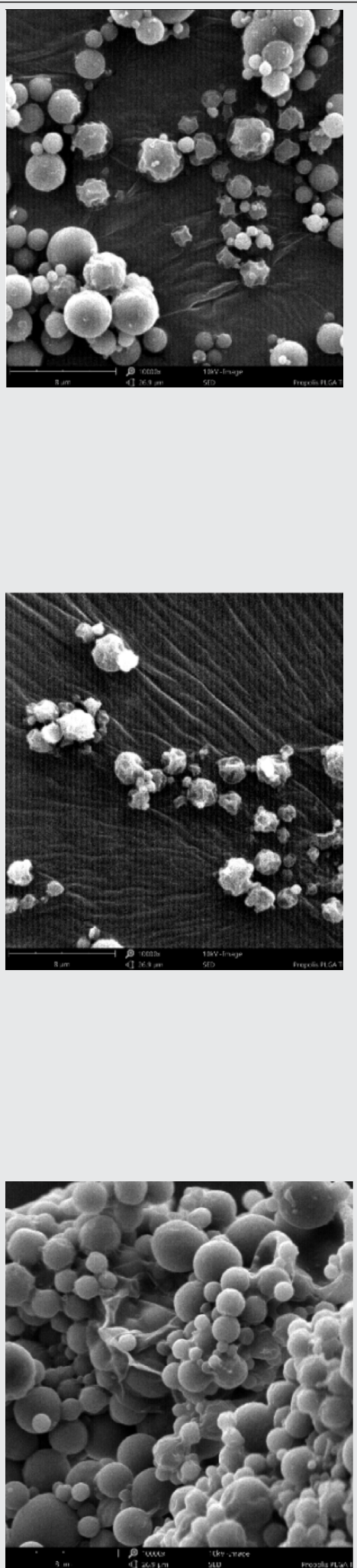


\section{- Table 3 (Continued)}

$\begin{array}{lll}\text { No. Pol. } & \text { PPR }^{\mathrm{a}} \quad \text { Amp. } 5000 \text { times }^{\mathrm{b}} \\ 4 & \mathrm{EC}\end{array}$
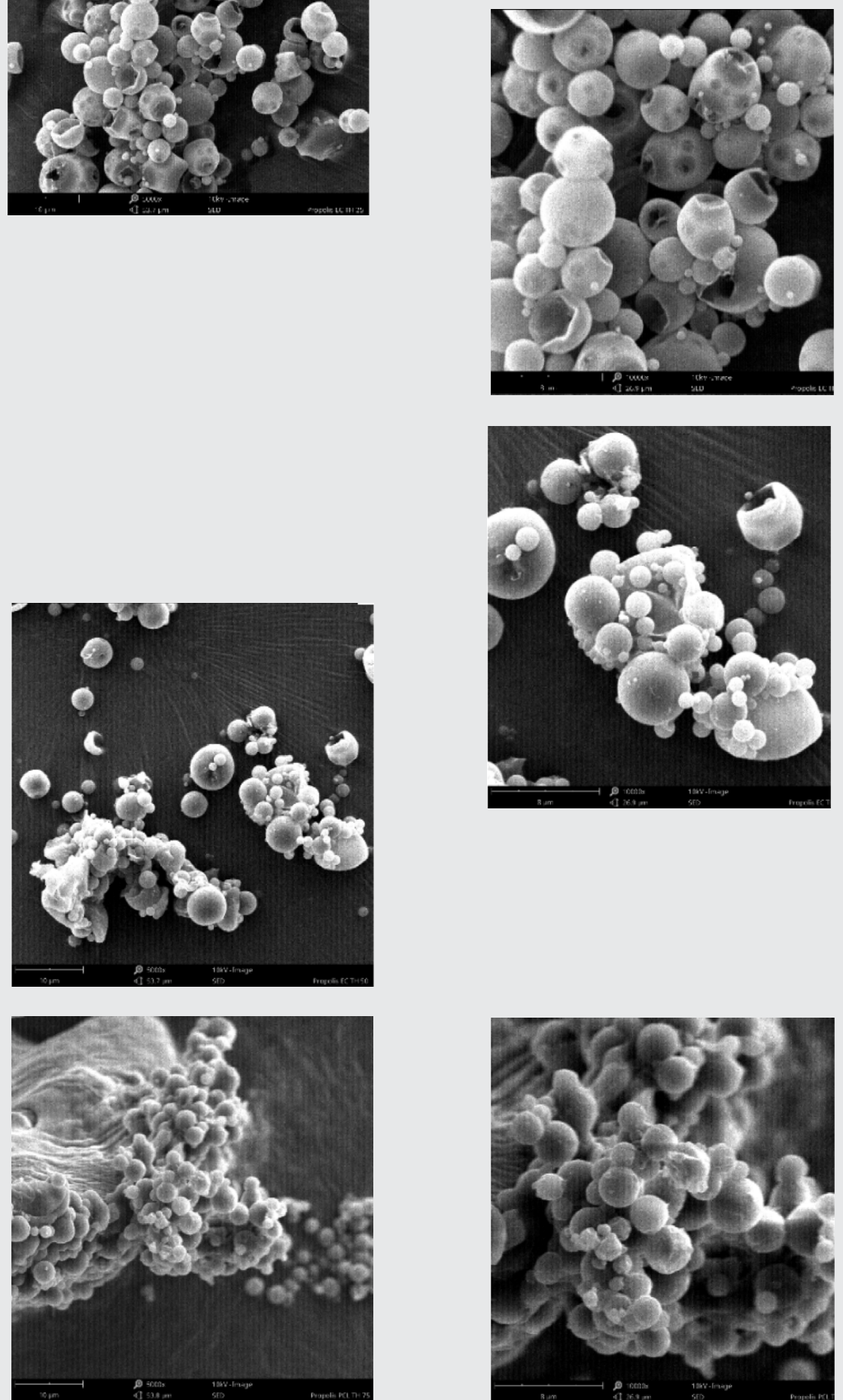


\section{- Table 3 (Continued)}

No.

Pol.

PCL

$1: 4$

8

$1: 2$
Amp. 5000 times $^{\mathrm{b}}$
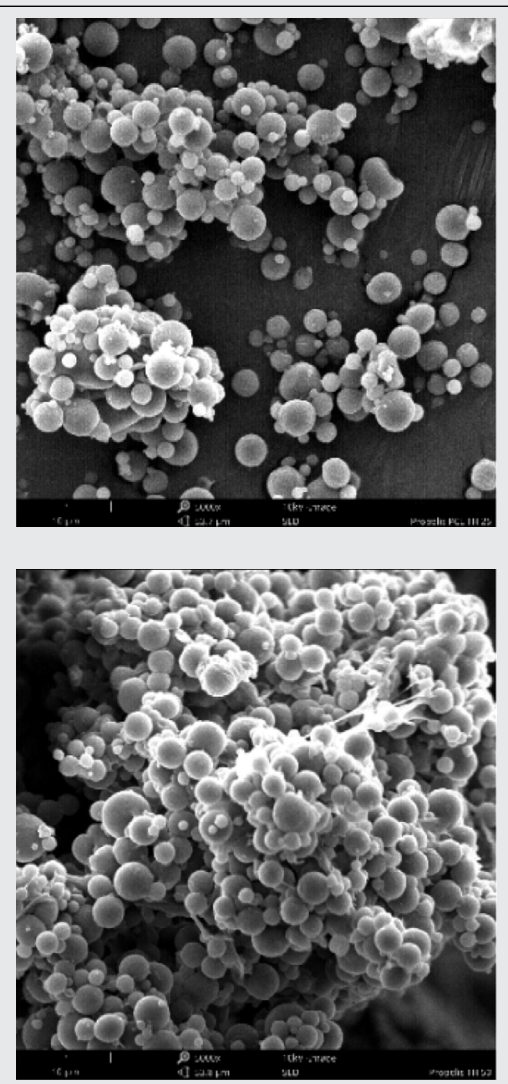

Amp. 10,000 times
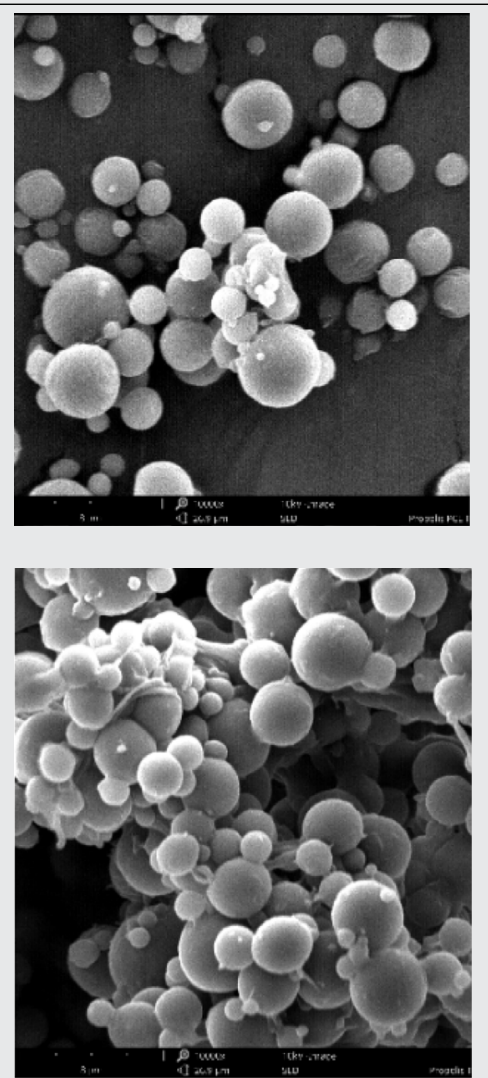


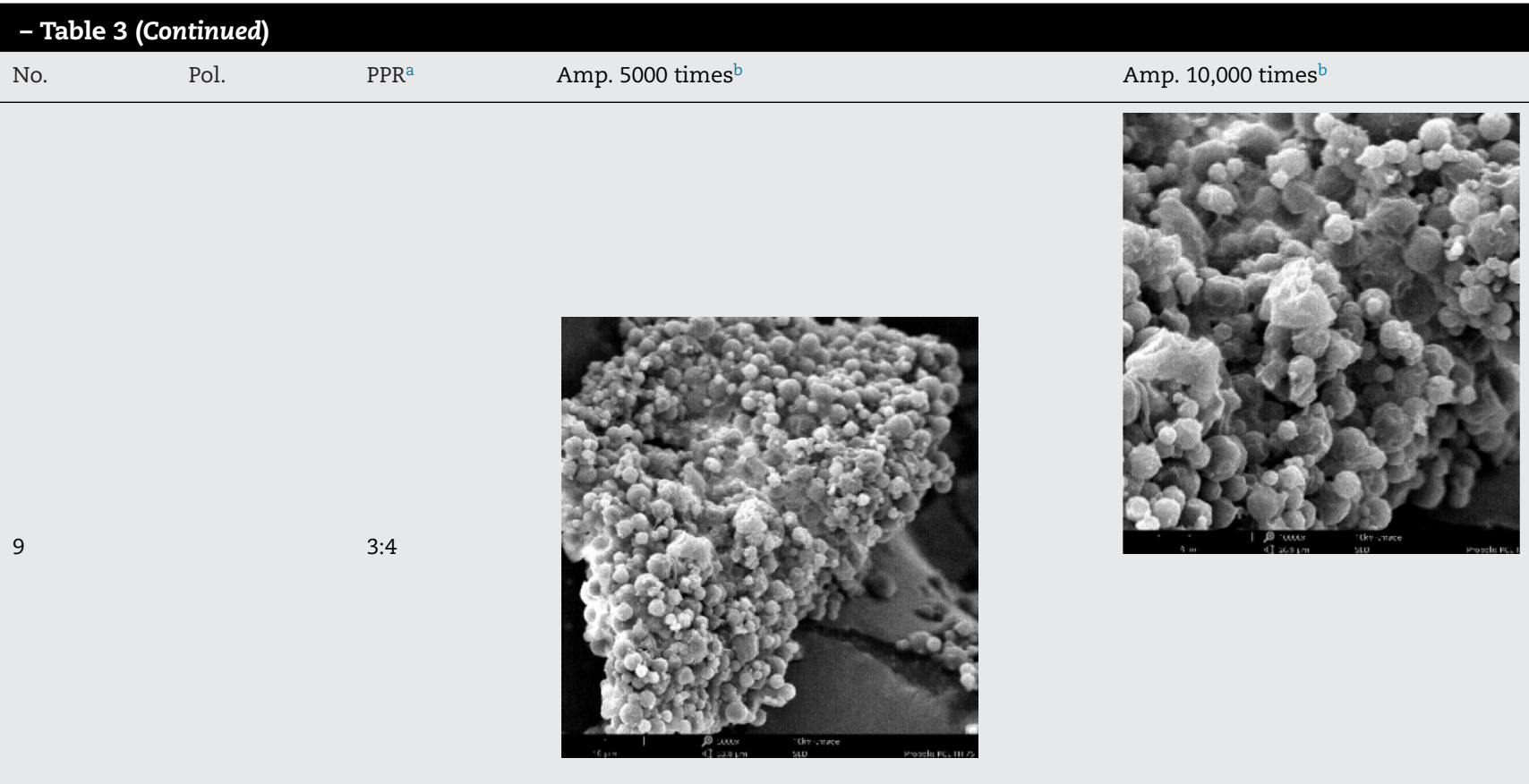

a PPR (w/w) - propolis extract to polymer ratio in a weight basis.

b Beam intensity of $10.00 \mathrm{kV}$ used in all micrographs.

Amp. - amplification; No. - entry number; Pol. - polymer; PPR - propolis to polymer ratio.

It can be concluded that propolis extracts-loaded microparticles present morphological characteristics dependent of the polymer carrier and the propolis extract to polymer ratio value.

\subsubsection{Assessment of the particle size distribution}

The graphical representation of the particle size distribution as well as the particle polydispersity index are presented in Fig. 2.

Regarding the results of the mean particle size, propolis extracts-loaded ethylcellulose microparticles demonstrated a mean particle size of $4.6 \pm 0.3 \mu \mathrm{m}$. In the case of propolis extracts-loaded poly(D,L-lactide-co-glycolide) and polycaprolactone microparticles, the particle sizes observed were $3.4 \pm 0.4$ and $3.3 \pm 0.5 \mu \mathrm{m}$, respectively (Fig. $2 \mathrm{~A}$ ). In all cases, the mean particle size seemed not to be affected by the propolis extract to polymer ratio as there were not observed significant differences among the experimental groups at the mean particle size as the P-values obtained were $0.405,0.627$ and 0.953 for propolis extracts-loaded ethylcellulose, poly(D,L-lactide-coglycolide) and polycaprolactone microparticles, respectively, considering a significant level of $95 \%$.

Concerning the results of the polydispersity index, it was verified a mean polydispersity index of $1.9 \pm 0.2,2.1 \pm 0.4$ and $2.3 \pm 0.5$ for propolis extracts-loaded ethylcellulose, poly(D,L-lactide-co-glycolide) and polycaprolactone microparticles, respectively. Similarly, to the results obtained for the mean particle size, the polydispersity index was not affected by the propolis extract to polymer ratio, as for all cases was verified a $p$-value higher than 0.050 , considering a significant level

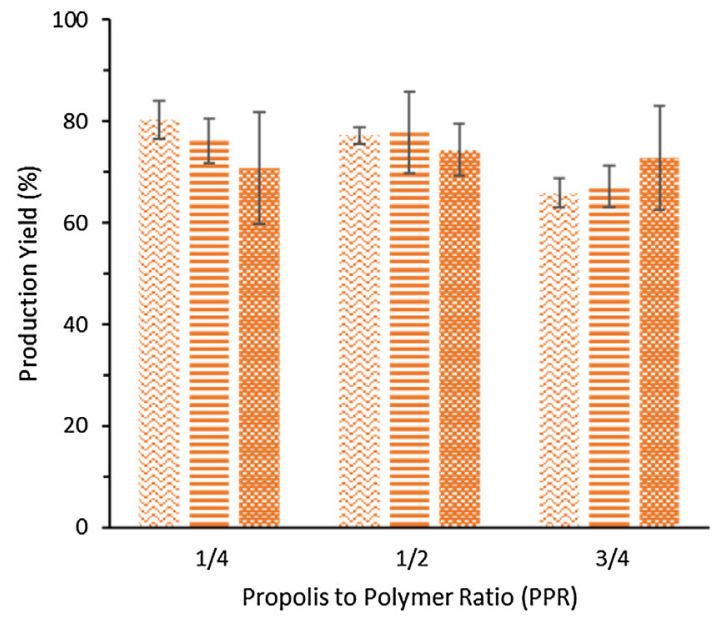

(A)

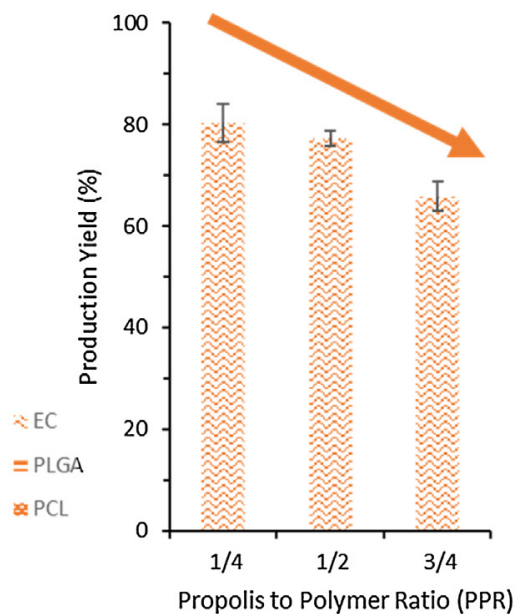

(B)

Fig. 1 - Results of propolis extracts-loaded microparticles production yield using different polymer-carriers and propolis extract to polymer ratios (A) and using only ethylcellulose as polymer carrier and different propolis extract to polymer ratios (B). 


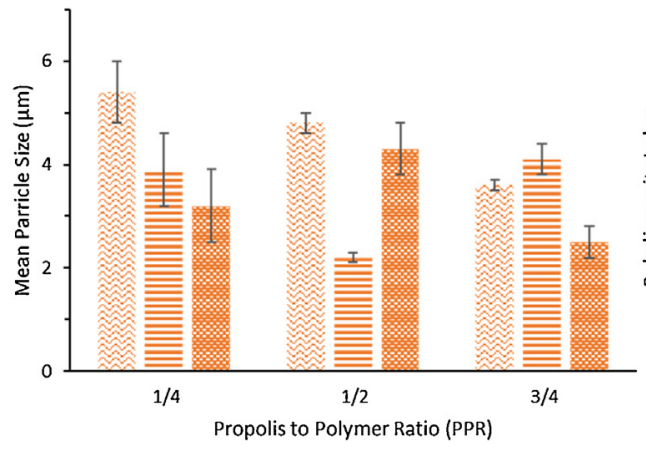

(A)

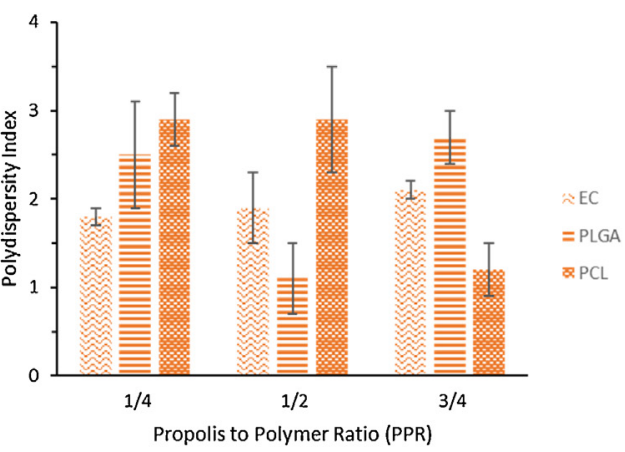

(B)

Fig. 2 - Results regarding the mean particle size (A) and the polydispersity index (B) of propolis extracts-loaded microparticles.

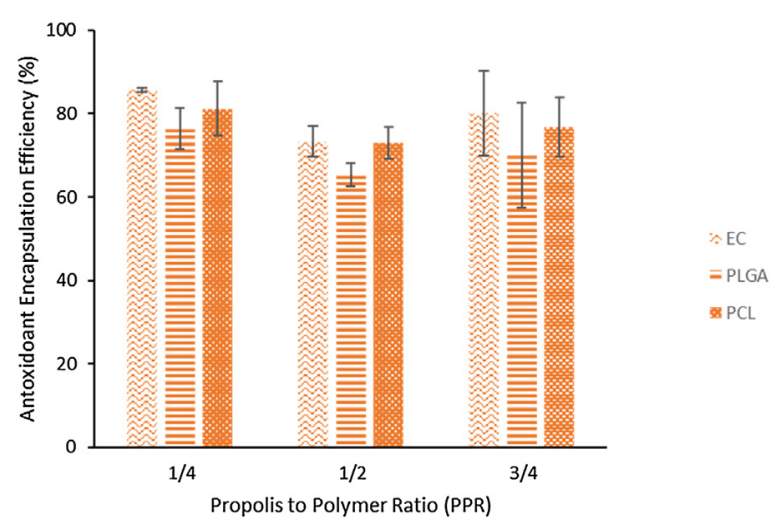

Fig. 3 - Antioxidant Encapsulation Efficiency of propolis extracts-loaded microparticles using different polymer-carriers and propolis extract to polymer ratios.

of $95 \%$ and therefore, no significative statistical difference in the polydispersity index were observed among the experimental groups (grouped by the propolis extract to polymer ratio).

The research presented by Avanço and Bruschi (2008) was focused on the microencapsulation of propolis extract in ethylcellulose microparticles, combining the emulsification and solvent evaporation approaches. The authors reported a mean particle size of $85.83 \mu \mathrm{m}, 18.7$ times fold higher than the mean particle size found for propolis extracts-loaded ethyl cellulose microparticles $(4.6 \pm 0.3 \mu \mathrm{m})$. Therefore, even though the results of this study indicate that the propolis extract to polymer ratio does not affect the mean particle size and a similar methodology for the obtainment of loaded microparticles was applied in both studies, it can be concluded that the stirring method, as well as the emulsifier concentration, may influence the mean particle size.

The authors Durán et al. (2007) applied similar approaches (emulsification and solvent evaporation) for the encapsulation of propolis extract in polycaprolactone. The authors reported that the mean particle size ranged from 5 to $10 \mu \mathrm{m}$. The obtained values by Durán et al. (2007) for the mean particle size were 1.5-3.0 times higher than the values obtained in the present study. Even though the results of both studies are entirely in agreement, it was confirmed that both formulation parameters and the experimental conditions defined for the study widely affect the results regarding the mean particle size.

In the present study it was observed that both the mean particle size as well as microparticles' polydispersity were not affected by the polymer type (EC, PLGA, PCL) neither by the propolis to polymer ratio. Probably, particles' size distribution is mainly dependent on the operational conditions (e.g., emulsification type, duration of emulsification, speed of emulsification, stirring speed time during solvent evaporation among others). The independence of the particles' size distribution from the polymer type as well as the polymer to bioactive compound ratio was also observed by Paulo and Santos (2020b).

To the authors best knowledge, there is a lack of published data regarding the study of polydispersity index of propolis extracts-loaded microparticles, suitable for a direct comparison.

3.3. Assessment the effect of the polymer carrier in the encapsulation of bioactive compounds in propolis extracts-loaded microparticles

\subsubsection{Quantitative assessment of encapsulation efficiency} of propolis extract-loaded microparticles

3.3.1.1. Assessment of the antioxidant encapsulation efficiency. The amount of antioxidants entrapped in ethylcellulose, poly(D,L-lactide-co-glycolide) and polycaprolactone polymerbased systems with different propolis extract to polymer ratios $(1 / 4,1 / 2$ and $3 / 4)$ was evaluated through the determination of the total non-enzymatic antioxidant capacity $\left(\mathrm{Cu}^{2+}\right.$ chelating method) and calculated using Eq. (3). The graphical representations of the total amount of antioxidants retained in microparticles polymer-based systems are shown in Fig. 3.

The mean antioxidant encapsulation efficiency ranged from $70.1 \pm 0.5 \%$ in the case of propolis extracts-loaded poly(D,L-lactide-co-glycolide) microparticles with a propolis extract to polymer ratio of $3 / 4$ and $85.7 \pm 0.5 \%$ in the case of propolis extracts-loaded polycaprolactone microparticles with a propolis extract to polymer ratio of $1 / 4$.

The mean antioxidant encapsulation efficiency of propolis extracts-loaded ethylcellulose microparticles was $79.7 \pm 7.7 \%$, corresponding to a total antioxidant loss of $20.3 \pm 7.7 \%$. The results showed that the antioxidant encapsulation efficiency using ethylcellulose was not affected by the propolis extract to polymer ratio as there was not observed differences among the three experimental groups (propolis extract to polymer ratio of $1 / 4,1 / 2$ and $3 / 4$ ), considering a significance level of $95 \%$ $(p=0.127>0.050)$.

In the case of using poly(D,L-lactide-co-glycolide) as a carrier material of propolis extract, the mean antioxidant encapsulation efficiency was $70.6 \pm 8.4 \%$, corresponding to a total antioxidant loss of $29.4 \pm 8.4 \%$. The null hypothesis of the propolis extract to polymer ratio does not influence the antiox- 
idant encapsulation efficiency was verified as the $p$-value was higher than $0.050(p=0.313)$, considering a significance of $95 \%$.

The mean antioxidant encapsulation efficiency of propolis extracts-loaded polycaprolactone microparticles was $77.0 \pm 6.3 \%$, corresponding to a total antioxidant loss of $20.3 \pm 7.7 \%$. The mean antioxidant encapsulation efficiency was not affected by the propolis extract to polymer ratio chosen for the study $(1 / 4,1 / 2$, or $3 / 4)$ as the $p$-value was higher than $0.050(p=0.305)$.

The total antioxidant capacity of the methanolic propolis extract was $2.7 \pm 0.6 \mathrm{mM}$ Trolox Equivalents, corresponding to $68.6 \pm 3.4 \mathrm{mg}$ Trolox Equivalents/g of propolis extract. This result is similar to that obtained by Hatano et al. (2012) where the antioxidant capacity ranged from $54.2 \pm 3.0 \mathrm{mg}$ (in red propolis samples from Cuba) to $90.9 \pm 0.6 \mathrm{mg}$ Trolox Equivalents/g (in Chinese samples).

Having into account the different encapsulation efficiencies, the total amount of antioxidants encapsulated in ethyl cellulose, poly(D,L-lactide-co-glycolide) and polycaprolactone microparticles was $54.7 \pm 0.3 \mathrm{mg}$ Trolox Equivalents $/ g$ of propolis extract, $48.4 \pm 0.3 \mathrm{mg}$ Trolox Equivalents/g of propolis extract and $52.8 \pm 0.2 \mathrm{mg}$ Trolox Equivalents/g of propolis extract, respectively.

There were no observed significant differences on the encapsulation efficiencies among the PPRs, for all polymers selected in the present study. differences on the encapsulation efficiencies among the PPRs, for all polymers selected in the present study. Nevertheless, for all PPRs, propolis-loaded EC microparticles exhibited the highest encapsulation efficiency of antioxidants. This observation may be related to capability of hydrophilic bioactive compounds present in propolis extract establish interactions with the ethylcellulose through hydrogen bond.

There are available alternative methodologies to determine the free radical scavenging capacity and therefore the antioxidant capacity as well as the antioxidant encapsulation efficiency. The free radical scavenging can be determined by the use of the 2,2-diphenyl-1-picrylhydrazyl (DPPH) radical, by the use of the 2,2' -azinobis(3-ethylbenzothiazoline-6-sulfonic acid) diammonium salt (ABTS) radical, by the oxygen radical absorbance capacity (ORAC) assay, by the superoxyde dismutase (SOD) assay, by the ferric reducing antioxidant potential (FRAP) assay, among others (Dudonné et al., 2009). The radical scavenging propensity can be, alternatively, evaluated through the formation of $\mathrm{Cu}^{2+}$ - antioxidants complexes of as described by Jabeen et al. (2017). As described in Section 2.4.1 of the present study, this alternative method was employed to determine the total content of antioxidants in propolis extracts-loaded microparticles.

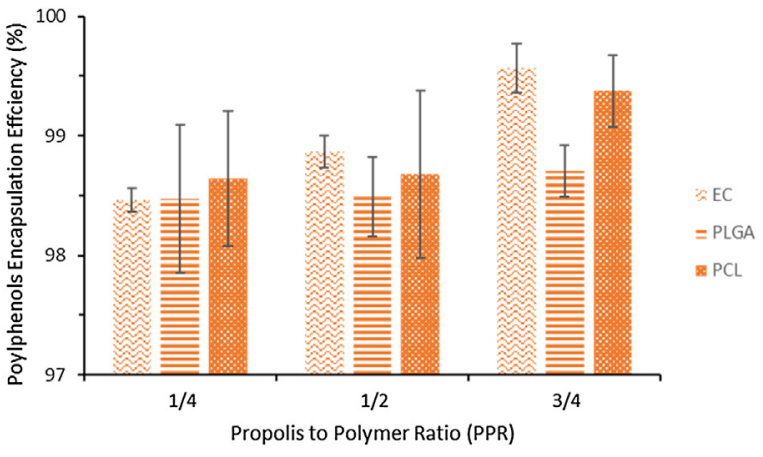

Fig. 4 - Polyphenols Encapsulation Efficiency of propolis extracts-loaded microparticles using different polymer-carriers and propolis extract to polymer ratios.

However, to the authors' best knowledge, this is the first time that is reported the use of this alternative method for the quantification of the total antioxidant content of propolis extracts and propolis extracts-loaded particles. Accordingly, there is a lack of published data suitable for direct comparison. Moreover, the majority of the published data found in the literature report the encapsulation efficiency considering only the content of phenolic compounds. Therefore, the quantitative assessment of encapsulation efficiency of propolis extracts-loaded microparticles was evaluated considering the content in phenolic compounds in propolis extracts as well as in propolis extracts-loaded microparticles.

3.3.1.2. Assessment of the polyphenolic encapsulation efficiency. In this study, the total amount of polyphenols encapsulated into ethylcellulose, poly(D,L-lactide-co-glycolide) and polycaprolactone polymeric systems with different propolis extract to polymer ratios $(1 / 4,1 / 2$ and $3 / 4)$ were evaluated considering the Eq. (5) and the determination of the total phenolic encapsulation efficiency as described in Section 2.4.2. The graphical representations of phenolic encapsulation efficiency for each polymer and propolis extract to polymer ratio are presented in Fig. 4. As far as the authors know there is no information available on the literature regarding the antioxidant encapsulation efficiency of propolis extractsloaded microparticles what hampers direct comparisons with the hereby reported results.

The mean phenolic encapsulation efficiency of propolis extracts-loaded ethyl cellulose microparticles was $99.0 \pm 1.0 \%$, corresponding to a phenolic loss of only $1.0 \pm 1.0 \%$. The phenolic encapsulation efficiency of microparticles formulated using ethylcellulose was not affected by the propolis extract to polymer ratios as was not observed statistically significant differences among the three experimental groups

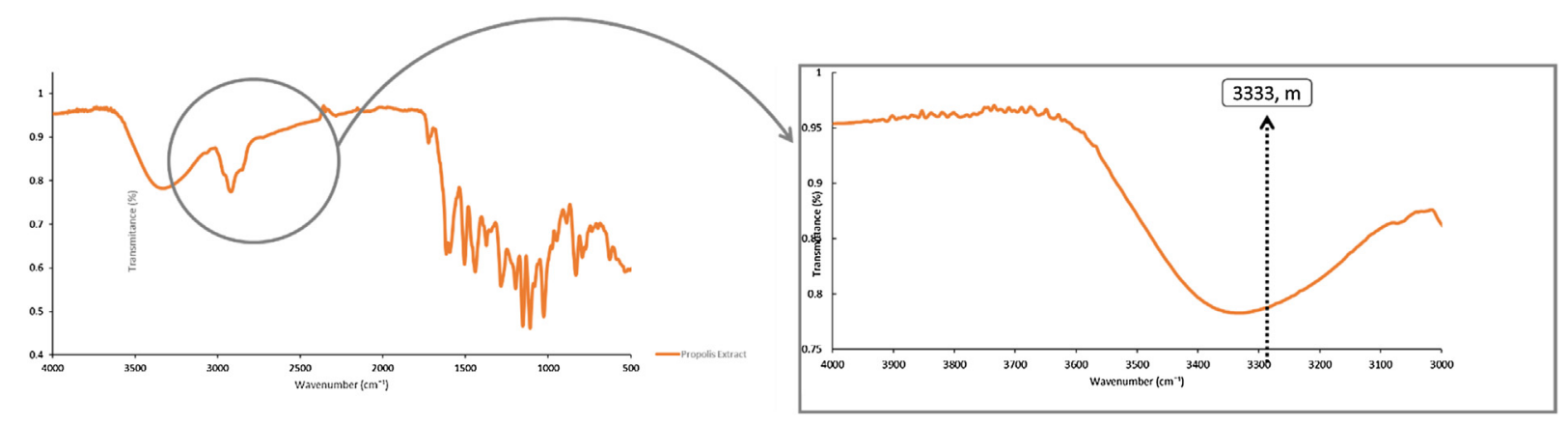

(A)

(B)

Fig. 5 - ATR-FTIR spectrum of propolis extract samples ((A) - non zoomed; (B) - zoomed between 4000 and $\left.3000 \mathrm{~cm}^{-1}\right)$. 
(propolis extract to polymer ratio of $1 / 4,1 / 2$ and $3 / 4$ ) as the $p$-value was higher than 0.050 considering a significance level of $95 \%(p=0.439)$. The mean amount of phenolic compounds encapsulated in ethyl cellulose microparticulated systems was $973.9 \pm 0.4 \mathrm{mg}$ gallic acid equivalents/g of propolis extract. Comparing the encapsulation efficiency of antioxidants and phenolic compounds into ethyl cellulose $(99.0 \pm 1.0 \%$ versus $79.7 \pm 7.7 \%)$, it can be stated that the majority of the incorporated antioxidants were phenolic compounds. Therefore, ethylcellulose can be considered an exceptional carrier for the inclusion of polyphenols present in propolis extracts.

The results regarding the phenolic encapsulation efficiency of propolis extracts-loaded poly(D,L-lactide-co-glycolide) microparticles point are similar to the ones obtained with propolis extracts-loaded ethyl cellulose microparticles. The mean encapsulation efficiency of phenolic compounds in poly(D,L-lactide-co-glycolide) polymer-based systems was $98.6 \pm 0.4 \%$, corresponding to a phenolic loss of $1.4 \pm 0.4 \%$. The encapsulation efficiency of phenolic compounds in this biocompatible polymer was not affected by the propolis extract to polymer ratio as, considering a confidence level of $95 \%$, the null hypothesis $\left(\mathrm{H}_{0}\right.$ : the phenolic encapsulation efficiency is not affected by the propolis extract to polymer ratio value) was verified $(p=0.766>0.050)$. As presented in the previous section (3.3.1.1), the mean antioxidant encapsulation efficiency using poly(D,L-lactide-co-glycolide) was $70.6 \pm 8.4 \%$, therefore as the phenolic encapsulation efficiency was higher than the obtained value for the antioxidant encapsulation efficiency, it can be concluded that poly(D,L-lactide-co-glycolide) is a suitable carrier for the incorporation of phenolic compounds present in propolis extracts in poly(D,L-lactide-co-glycolide)based microparticles rather than other types of antioxidants. The mean total amount of phenolic compounds encapsulated in poly(D,L-lactide-co-glycolide)-based microparticles was $978.9 \pm 0.4 \mathrm{mg}$ gallic acid equivalents/g of propolis extract.

The mean phenolic encapsulation efficiency of propolis extracts-loaded polycaprolactone microparticles was $98.9 \pm 0.6 \%$, corresponding to the total phenolic loss of $1.1 \pm 0.6 \%$. The phenolic encapsulation efficiency was not affected by the propolis extract to polymer ratio as the $p$-value obtained was higher than 0.050 , considering a significance level of $95 \%(p=0.256)$. Like the results obtained for the phenolic and antioxidant encapsulation efficiency of propolis extracts-loaded ethylcellulose and poly(D,L-lactideco-glycolide) microparticles, the encapsulation efficiency of phenolic compounds was higher than the encapsulation efficiency of antioxidants (antioxidant encapsulation efficiency of $77.0 \pm 6.4 \%$, for loaded polycaprolactone particles). Therefore, the chosen polymer carrier - polycaprolactone - can be considered a leading carrier for the incorporation and protection of phenolic compounds present in propolis extracts. The total amount of phenolic compounds incorporated in polycaprolactone polymeric based microsystems was $990.1 \pm 0.3 \mathrm{mg}$ of gallic acid equivalents/g of propolis extract.

It is essential to highlight that the total amount of phenolic compounds of the studied red propolis samples and, consequently, that incorporated in the different microsystems is very superior to that reported in other studies using red propolis from other geographical origins. Indeed, in a study performed by Hatano et al. (2012) the total polyphenol content of the ethanolic extracts was $433.8 \pm 1.7$ (in Chinese red propolis), $292.7 \pm 2.9$ (in samples from Brazil) and $127.3 \pm 2.7 \mathrm{mg}$ gallic acid equivalents/g of propolis (in samples from Cuba).
The total polyphenol content of our samples is also superior to that reported by Machado et al. (2016), who studied Brazilian red propolis extracts obtained using both ethanol and supercritical fluid procedures. These differences may be due to the botanical and geographical origins of the samples. Notwithstanding, we believe that the superior concentrations of total phenolics obtained in this study confirm that methanolic propolis extracts present higher amounts of biologic compounds in comparison with extracts obtained using other solvents (Karapetsas et al., 2019).

Independent of the polymer carrier, the propolis extract to polymer ratio seemed not to affect the encapsulation of phenolic compounds. The results presented in Table 4 are presented by the maximum and the minimum value that was obtained for the antioxidant encapsulation efficiency (\%), phenolic compounds encapsulation efficiency (\%) and total phenolic content (in $\mathrm{mg}$ of gallic acid equivalents/g of propolis extract). The maximum and the minimum phenolic compounds encapsulation efficiency was obtained using ethyl cellulose as the polymer carrier. However, this polymer carrier shown to be most efficient for the incorporation of antioxidants as both the maximum and the minimum values obtained for the antioxidant encapsulation efficiency was the highest when compared with the other two polymers. The minimal values for both the maximum and minimum level for the total phenolic compounds content were the lowest. Therefore, it can be concluded that ethylcellulose is an exceptional polymer carrier for the incorporation of antioxidants present in propolis extracts. However, this polymer has shown not to be the most suitable one for the incorporation of phenolic compounds.

The antioxidant encapsulation efficiency of propolis extracts-loaded polycaprolactone microparticles presented the mid-values for both levels (maximum and minimum levels). However, regarding the results of the phenolic compounds encapsulation efficiency, for this polymer, the maximum value at the minimum level was found to be the highest (98.3\%), and the maximum value for the maximum level was close to the result obtained with ethyl cellulose (99.5\%). Moreover, polycaprolactone showed to strongly incorporate phenolic compounds as both for the maximum, and minimum levels the total phenolic compounds were the highest found ( $989.8 \mathrm{mg}$ gallic acid equivalents/g of propolis extract for the minimum level and $990.4 \mathrm{mg}$ gallic acid equivalents/g of propolis extract for the maximum level). Therefore, it can be concluded that polycaprolactone, shown to be the best polymer for the incorporation and protection of phenolic compounds present in propolis extracts.

The authors Zhang et al. (2018) studied the antioxidant activity and in vitro release of propolis extract by acid-induced aggregation using heat-denatured zein and carboxymethyl chitosan. They found the optimal encapsulation efficiency based on the total phenolic content was $83.36 \%$, corresponding to a formulation of $40 \%$ of propolis extract loading, zein to carboxymethyl chitosan ratio of $1 / 1(\mathrm{w} / \mathrm{w})$ and carboxymethyl chitosan to $\mathrm{Ca}^{2+}$ ratio of 50/1 (w/w). Comparing the results obtained by Zhang et al. (2018), the obtained results in this study, were quite improved - the encapsulation efficiencies found (based on the total amount of phenolic compounds) were higher for all the polymers studied.

Propolis extracts-loaded pea protein microparticles were produced by Jansen-alves et al. (2019) and the encapsulation efficiency of loaded microparticles was evaluated based on the amount of phenolic compounds incorporated. The 
Table 4 - Results regarding the maximum and the minimum levels found for the antioxidant, phenolic encapsulation efficiency and the total phenolic content.

\begin{tabular}{|c|c|c|c|c|c|c|}
\hline \multirow[t]{2}{*}{ Polymer } & \multicolumn{2}{|c|}{ TAEE (\%) } & \multicolumn{2}{|c|}{ TPEE (\%) } & \multicolumn{2}{|c|}{ TPC (mg of GAE/g of propolis extract) } \\
\hline & Min & Max & Min & Max & Min & $\operatorname{Max}$ \\
\hline EC & 72.0 & 87.4 & 98.0 & 100.0 & 973.5 & 974.3 \\
\hline PLGA & 62.2 & 79.0 & 98.2 & 99.0 & 978.5 & 979.3 \\
\hline PCL & 70.6 & 83.4 & 98.3 & 99.5 & 989.8 & 990.4 \\
\hline
\end{tabular}

authors found that the maximum value for the encapsulation efficiency was $97.00 \pm 0.18 \%$ with a carrier concentration of $2 \%$ and a propolis extract concentration of $5 \%$. Even though many factors are dissimilar to the ones selected for this study (including the encapsulation technique), the maximum value found was even lower than the values found for this parameter in the present study.

However, it was verified a lack of studies for the direct comparison of phenolic compounds encapsulation efficiency. Nevertheless, the obtained results are promising as, independent of the carrier, and the polymer to propolis extract ratio, the encapsulation efficiency of phenolic compounds remained above $95 \%$.

\subsubsection{Qualitative assessment of effective encapsulation of} propolis- extract into microparticulated systems

A qualitative assessment of the effectiveness of the encapsulation of propolis extract in ethylcellulose, poly(D,L-lactideco-glycolide) and polycaprolactone polymer-based systems was evaluated by the Fourier transform infrared spectroscopy (FTIR). This technique can be employed to identify the presence of specific functional groups in the propolis extract sample and to evaluate if they are present in the surface of loaded microparticles. More accurately, the use of the FTIR mode - the Attenuated Total Reflectance (ATR) mode allows probing surface properties of the materials rather than their bulk properties. Therefore, the FTIR-ATR was employed to identify specific chemical functional groups present in propolis extract in the surface of propolis extracts-loaded microparticles to evaluate the effectiveness of the incorporation of this extract in different polymeric matrices.

The FTIR spectrum of propolis extract used in this study is presented in Fig. 5 (A - full spectrum; B - zoomed spectrum). The bands present in the $2000-500 \mathrm{~cm}^{-1}$ spectrum area correspond probably to an intense and high quantity of vibrational modes of different chemical bonds present in propolis extract. In this spectrum area bands overlap may occur. Therefore, a clean area of the propolis FTIR spectrum was chosen for an accurate evaluation of the effectiveness of the incorporation of propolis extract into polymer-based systems (Fig. 5B). The bands associated with the $\mathrm{O}-\mathrm{H}$ groups stretching vibrational mode of propolis extract molecules are shown at $3333 \mathrm{~cm}^{-1}$ (medium intensity). A previous study on encapsulation, antioxidant activity and in vitro release of propolis extract formulated by acid-induced aggregation using heat-denatured zein and carboxymethyl chitosan obtained a FTIR band at $3413 \mathrm{~cm}^{-1}$ (Zhang et al., 2018). These authors attributed this band to the $\mathrm{O}-\mathrm{H}$ stretching vibration mode of hydroxyl groups that may belong to phenolic compounds and carbohydrates. Another study regarding the microencapsulation of propolis extract in a protein matrix by spray-drying, found a spectra broadband between 3560 and $3100 \mathrm{~cm}^{-1}$
(Jansen-alves et al., 2018). These authors assigned this region to the $\mathrm{O}-\mathrm{H}$ stretching of hydroxyl groups present in phenolic compounds. The values found by us are similar to those reported in this later study.

In addition, it should be stated that many differences among propolis extracts samples may be found, as the geographical location of propolis may strongly affect its chemical composition. Moreover, other relevant factors as the extraction approach, solvents intrinsic characteristics and solvent ratio may substantially affect the final chemical composition of the obtained propolis extract sample (Ahangari et al., 2018; Silva et al., 2012). These factors may be the explanation for the differences in the wavenumber assigned to the $\mathrm{O}-\mathrm{H}$ groups stretching vibrational mode of phenolic compounds present in the extract found to be at a wavenumber of $3333 \mathrm{~cm}^{-1}$.

Fig. 6 depicts the FTIR spectra for propolis extractsloaded microparticles from 4000 to $3000 \mathrm{~cm}^{-1}$ (A - FTIR spectra obtained for propolis extract ethyl cellulose loaded microparticles; B - FTIR spectra obtained for propolis extract poly(D,L-lactide-co-glycolide) loaded microparticles; C - FTIR spectra obtained for propolis extract polycaprolactone loaded microparticles).

The signature sharp peak of propolis extract at $3333 \mathrm{~cm}^{-1}$ is absence of propolis extracts-loaded ethylcellulose microparticles (Fig. 6A). It was verified that when propolis was formulated in ethylcellulose microparticles, they present their signature sharp peak associated to the $\mathrm{O}-\mathrm{H}$ groups stretching vibrational modes at $3457 \mathrm{~cm}^{-1}$ in the case of propolis-loaded ethyl cellulose microparticles formulated considering a propolis to polymer ratio of $1 / 4$ and $1 / 2$, and at a wavenumber of $3490 \mathrm{~cm}^{-1}$ in the case of microparticles formulated considering a propolis to polymer ratio of $3 / 4$. As reported by Paulo and Santos (2018a,b), polymer only microparticles formulated in same conditions as reported in this study, present a signature broad $\mathrm{O}-\mathrm{H}$ stretching vibrational band at $3464 \mathrm{~cm}^{-1}$. According, the energy of the $\mathrm{O}-\mathrm{H}$ stretching vibration was similar in the case of propolis extracts-loaded ethyl cellulose microparticles formulated with a propolis extract to polymer ratio of $1 / 4,1 / 2$ and $3 / 4$ as no significative shifts were observed in all the cases $-7,7$ and $33 \mathrm{~cm}^{-1}$ in the case of microparticles formulated with $1 / 4,1 / 2$ and $3 / 4$, respectively.

Regarding the FTIR results of propolis extracts-loaded poly(D,L-lactide-co-glycolide) microparticles, the signature peak of propolis extract at $3333 \mathrm{~cm}^{-1}$ was not found in microparticles formulated with poly(D,L-lactide-co-glycolide). Both propolis extracts-loaded poly(D,L-lactide-co-glycolide) microparticles with a propolis extract to polymer ratio of $1 / 4$ or $1 / 2$ presented a weak $\mathrm{O}-\mathrm{H}$ group stretching vibrational band at $3483 \mathrm{~cm}^{-1}$. In the case of propolis-extract loaded polycaprolactone microparticles formulated with a propolis extract to polymer ratio of $3 / 4$, the FITR spectrum presented a broad weak $\mathrm{O}-\mathrm{H}$ group stretching vibrational peak at 


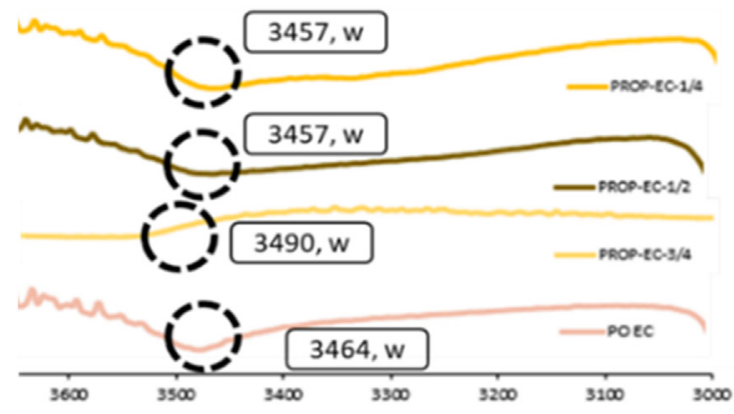

(A)

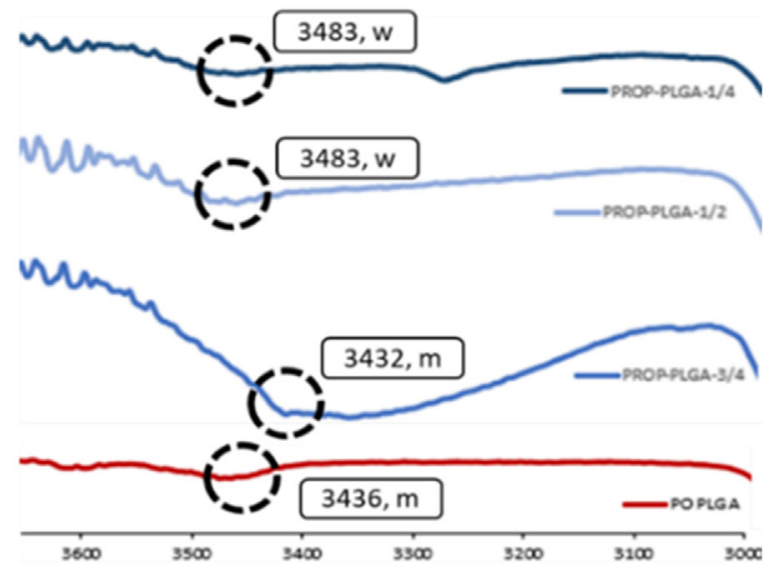

(B)

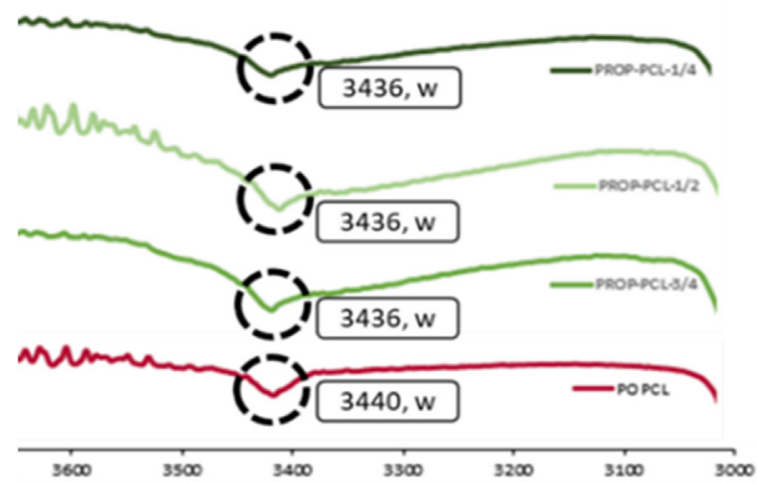

(C)

Fig. 6 - ATR-FTIR spectra of propolis extracts-loaded ethyl cellulose (A), poly(D,L-lactide-co-glycolide) (B) and PCL (C) microparticles with different propolis extract to polymer ratios as well as the corresponding polymer-only microparticles.

$3432 \mathrm{~cm}^{-1}$. In this study it was also found that the $\mathrm{O}-\mathrm{H}$ groups stretching vibrational peak of poly(D,L-lactide-co-glycolide) only microparticles appears at $3436 \mathrm{~cm}^{-1}$. The results of this study corroborate the hypothesis of efficient incorporation of propolis extract into poly(D,L-lactide-co-glycolide) polymerbased microparticulate systems, as the energy associated $\mathrm{O}-\mathrm{H}$ groups stretching vibrational mode of loaded microparticles was similar to polymer only microparticles - corresponding to non-significative shifts of 47,47 , and $4 \mathrm{~cm}^{-1}$.

Similarly to the FTIR studies of propolis extractsloaded microparticles formulated with ethyl cellulose and poly(D,L-lactide-co-glycolide), the FTIR spectra of propolis extracts-loaded microparticles formulated with polycaprolactone did not present the signature peak of propolis extract at $3333 \mathrm{~cm}^{-1}$. The FTIR spectra of propolis extracts-loaded polycaprolactone microparticles presented a weak $\mathrm{O}-\mathrm{H}$ group stretching vibrational peak at $3436 \mathrm{~cm}^{-1}$ shifting only $4 \mathrm{~cm}^{-1}$ of the peak assigned for the $\mathrm{O}-\mathrm{H}$ groups stretching vibrational mode of hydroxyl groups in polymer only microparticles formulated with the PCL polymer (peak at $3440 \mathrm{~cm}^{-1}$ ).

We believe that the results obtained in this study are promising. The signature $\mathrm{O}-\mathrm{H}$ groups stretching vibrational peak at $3333 \mathrm{~cm}^{-1}$, present in the propolis extracts, was not detected in any of the evaluated spectra and no significant shifts were observed in comparison to the signature band present in microparticles formulated only with polymer Therefore, an efficient incorporation of propolis extracts was obtained for all the used polymers and for all assessed propolis extract to polymer ratios.

\section{Conclusions}

The raised hypothesis was verified: propolis extract was successfully microencapsulated in lipophilic polymer-carriers - ethylcellulose, polycaprolactone, and poly(D,L-lactide-coglycolic acid) - at three different polymer to propolis extract ratios - 1/4, $1 / 2$ and $3 / 4$ - by double emulsion solvent evaporation approach. The physicochemical characterization of propolis extracts-loaded microparticles was performed regarding the product yield, external morphology, particle size distribution, and particle polydispersity. The obtained microparticles presented similar external morphological characteristics and particle size distribution, being independent of the polymer to propolis extract ratio. Slight differences were observed among microparticles produced with different polymer-carriers. The product yield was much improved over other techniques reported in the literature for propolis encapsulation, such as spray drying.

Furthermore, a quantitative and qualitative analysis of the encapsulation efficiency was performed. The quantitative evaluation was carried out considering the antioxidant encapsulation and phenolic compounds encapsulation efficiencies. The obtained results are remarkable as the phenolic compounds encapsulation efficiency was higher than $98.0 \%$. The encapsulation efficiencies were shown to be independent of the propolis extract to polymer ratio, for the polymer used in this study. The polymer carrier, ethylcellulose revealed excellent properties for the general encapsulation of antioxidants $(79.7 \pm 7.7 \%)$ whereas the polycaprolactone polymer-based microsystems seemed to be the best polymer for the entrapment and protection of phenolic compounds present in propolis extract samples (mean phenolic compounds encapsulation efficiency of propolis extracts-loaded polycaprolactone microparticles of $98.9 \pm 0.6 \%$ ).

The FTIR results demonstrated that the propolis extracts were efficient incorporated into polymer carriers as the signature $\mathrm{O}-\mathrm{H}$ groups stretching vibrational peak at $3333 \mathrm{~cm}^{-1}$ presents in the propolis extract samples were not observed in any of FTIR spectra of loaded microparticles.

This study on the microencapsulation of propolis extracts by double emulsion solvent evaporation technique provide a promising and feasible microencapsulation approach and polymer-carriers for the application of propolis extractsloaded microparticles in functional matrices, like foods. 


\section{Conflict of interest}

Authors declare no conflict of interest or any financial benefit from direct or indirect applications of the research.

\section{Declaration of Competing Interest}

The authors report no declarations of interest.

\section{Acknowledgments}

This work was financially supported by project UIDB/00511/2020 - Laboratory for Process Engineering, Environment, Biotechnology, and Energy - LEPABE funded by national funds through FCT/MCTES (PIDDAC). This work was developed under the doctoral program in Chemical and Biological Engineering (PDEQB), financially supported by the grant NORTE-08-5369-FSE-000028, co-financed by the Northern Regional Operational Program (NORTE 2020) through Portugal 2020 and the European Social Fund (ESF).

\section{References}

Ahangari, Z., Naseri, M., Vatandoost, F., 2018. Propolis: chemical composition and its applications in endodontics. Iran. Endod. J. 13, 285-292, http://dx.doi.org/10.22037/iej.v13i3.20994.

Ankovaa, V.S.B., Astrob, S.L.D.E.C., Arcuccic, M.C.M., 2000. Propolis: recent advances in chemistry and plant origin. Apidologie 31, 3-15.

Avanço, G.B., Braschi, M.L., 2008. Preparation and characterisation of ethylcellulose microparticles containing propolis. Rev. Ciencias Farm. Basica Apl. 29, 129-134.

Avanço, G.B., Bruschi, M.L., 2008. Preparation and Characterisation of Ethylcellulose Microparticles Containing Propolis.

Ballesteros, L.F., Ramirez, M.J., Orrego, C.E., Teixeira, J.A., Mussatto, S.I., 2017. Encapsulation of antioxidant phenolic compounds extracted from spent coffee grounds by freeze-drying and spray-drying using different coating materials. Food Chem. 237, 623-631, http://dx.doi.org/10.1016/j.foodchem.2017.05.142.

Bankova, V., 2005. Chemical diversity of propolis and the problem of standardization. J. Ethnopharmacol 100, 114-117, http://dx.doi.org/10.1016/j.jep.2005.05.004.

Bodini, R.B., Sobral, P.J.A., Carvalho, R.A., 2013. Properties of gelatin-based films with added ethanol e propolis extract. LWT - Food Sci. Technol. 51, 104-110, http://dx.doi.org/10.1016/j.lwt.2012.10.013.

Bruschi, M.L., Cardoso, M.L.C., Lucchesi, M.B., Gremião, M.P.D., 2003. Gelatin microparticles containing propolis obtained by spray-drying technique: preparation and characterization. Int. J. Pharmaceut. 264, 45-55, http://dx.doi.org/10.1016/S0378-5173(03)00386-7.

Busch, M., Kolender, A.A., Santagapita, P.R., Buera, M.P., 2015. Vinal gum, a galactomannan from Prosopis ruscifolia seeds: physicochemical characterization. Food Hydrocoll. 51, http://dx.doi.org/10.1016/j.foodhyd.2015.04.035.

Busch, V.M., Pereyra-Gonzalez, A., Segatin, N., Santagapita, P.R., Poklar Ulrih, N., Buera, M.P., 2017. Propolis encapsulation by spray drying: characterization and stability. LWT - Food Sci. Technol. 75, http://dx.doi.org/10.1016/j.lwt.2016.08.055.

Carvalho, A.S., Silva, J., Ho, P., Teixeira, P., Malcata, F.X., Gibbs, P., 2004. Effects of various sugars added to growth and drying media upon thermotolerance and survival throughout storage of freeze-dried Lactobacillus delbrueckii ssp. Bulgaricus. Biotechnol. Prog., 248-254.

Catchpole, O., Mitchell, K., Bloor, S., Davis, P., Suddes, A., 2018. Anti-gastrointestinal cancer activity of cyclodextrin-encapsulated propolis. J. Funct. Foods 41, 1-8, http://dx.doi.org/10.1016/j.jff.2017.12.023.

Cheng, H., Qin, Z.H., Guo, X.F., Hu, X.S., Wu, J.H., 2013. Geographical origin identification of propolis using GC - MS and electronic nose combined with principal component analysis. Food Res. Int. 51, 813-822, http://dx.doi.org/10.1016/j.foodres.2013.01.053.

Da Silva, F.C., Da Fonseca, C.R., De Alencar, S.M., Thomazini, M., Balieiro, J.C.D.C., Pittia, P., Favaro-Trindade, C.S., 2013. Assessment of production efficiency, physicochemical properties and storage stability of spray-dried propolis, a natural food additive, using gum Arabic and OSA starch-based carrier systems. Food Bioprod. Process. 91, 28-36, http://dx.doi.org/10.1016/j.fbp.2012.08.006.

Dangui, L., De Fl, J., Salvador, M., Plata-oviedo, V., Augusto, R., Tavares, W., Teresinha, S., 2017. Physico-chemical characteristics of microencapsulated propolis co-product extract and its effect on storage stability of burger meat during storage at -15 C. LWT - Food Sci. Technol. 76, 306-313, http://dx.doi.org/10.1016/j.lwt.2016.05.033.

Daugsch, A., Moraes, C.S., Fort, P., Park, Y.K., 2008. Brazilian Red Propolis - Chemical Composition and Botanical Origin 5, 435-441, http://dx.doi.org/10.1093/ecam/nem057.

Dota, D., Edilaine, M., Consolaro, L., Inez, T., Svidzinski, E., Bruschi, M.L., 2011. Antifungal Activity of Brazilian Propolis Microparticles Against Yeasts Isolated From Vulvovaginal Candidiasis., http://dx.doi.org/10.1093/ecam/neq029.

Dudonné, S., Vitract, X.C., Woillez, P., Marion Mérillon, J.-M., 2009. Comparative study of antioxidant properties and total phenolic content of 30 plant extracts of industrial interest using DPPH, ABTS, FRAP, SOD, and ORAC assays, 1768-1774.

Durán, N., Marcato, P.D., Buffo, C.M.S., Azevedo, M.M.M., Esposito, E., 2007. Poly ( $\varepsilon$-caprolactone)/propolis extract: microencapsulation and antibacterial activity evaluation. Pharmazie 62, 287-290.

Ely, M.R., Moura, S., 2017. Asian Pacif. J. Trop. Biomed. 7, 591-598, http://dx.doi.org/10.1016/j.apjtb.2017.06.009.

Freires, I.A., De Alencar, S.M., Rosalen, P.L., 2016. A pharmacological perspective on the use of Brazilian Red Propolis and its isolated compounds against human diseases. Eur. J. Med. Chem. 110, 267-279, http://dx.doi.org/10.1016/j.ejmech.2016.01.033.

Gomes, C.C., Barbosa, S., Ferreira, D.S., Rafael, B., Dias, D.A., Obrego, C.S., Silva, J., Godoy, R., Inez, T., Svidzinski, E., De Arau, R.R., Bruschi, M.L., 2014. Microparticles containing propolis and metronidazole: in vitro characterization, release study and antimicrobial activity against periodontal pathogens. Pharm. Dev. Technol. 7450, 173-180, http://dx.doi.org/10.3109/10837450.2013.763262.

Gómez-Caravaca, A.M., Gómez-Romero, M., Arráez-Román, D., Segura-Carretero, A., Fernández-Gutiérrez, A., 2006. Advances in the analysis of phenolic compounds in products derived from bees. J. Pharmaceut. Biomed. Anal. 41, 1220-1234, http://dx.doi.org/10.1016/j.jpba.2006.03.002.

González-búrquez, M.D.J., González-díaz, F.R., García-tovar, C.G., Carrillo-miranda, L., Soto-zárate, C.I., Canales-martínez, M.M., Penieres-carrillo, J.G., Crúz-sánchez, T.A., Fonseca-coronado, S., 2018. Comparison Between In Vitro Antiviral Effect of Mexican Propolis and Three Commercial Flavonoids Against Canine Distemper Virus., pp. 2018.

Han, F.Y., Thurecht, K.J., Whittaker, A.K., Smith, M.T., 2016. Bioerodable PLGA-based microparticles for producing sustained-release drug formulations and strategies for improving drug loading. Front. Pharmacol. 7, 1-11, http://dx.doi.org/10.3389/fphar.2016.00185.

Hatano, A., Nonaka, T., Yoshino, M., Ahn, M.-R., Tazawa, S., Araki, Y., Kumazawa, S., 2012. Antioxidant activity and phenolic constituents of Red Propolis from Shandong, China. Food Sci. Technol. Res. 18, 577-584.

Jabeen, E., Kausar, N., Ahmed, S., Murtaza, I., Ali, T., Hameed, S., 2017. Radical scavenging propensity of $\mathrm{Cu} 2+$, Fe 3+ complexes of flavonoids and in-vivo radical scavenging by $\mathrm{Fe}$ 3+-primuletin. Spectrochim. Acta Part A: Mol. Biomol. 
Spectrosc. 171, 432-438,

https://doi.org/10.1016/j.saa.2016.08.035

Jansen-alves, C., Fernandes, K.F., Crizel-cardozo, M.M., Krumreich, F.D., Borges, C.D., Zambiazi, R.C., 2018.

Microencapsulation of propolis in protein matrix using spray drying for application in food systems. Food Bioprocess Technol., 1422-1436.

Jansen-alves, C., Maia, D.S.V., Krumreich, F.D., Crizel-cardoso, M.M., Fioravante, J.B., Wladimir, P., Borges, C.D., Zambiazi, R.C., 2019. Propolis microparticles produced with pea protein: characterization and evaluation of antioxidant and antimicrobial activities. Food Hydrocoll. 87, 703-711, https://doi.org/10.1016/j.foodhyd.2018.09.004.

Jansen-alves, C., Zambiazi, R.C., 2019. Production of Propolis Extract Microparticles with Concentrated Pea Protein for Application in Food.

Karapetsas, A., Voulgaridou, G., Konialis, M., 2019. Propolis Extracts Inhibit UV-Induced Photodamage in Human Experimental In Vitro Skin Models.

Kubiliene, L., Laugaliene, V., Pavilonis, A., Maruska, A., Majiene, D., Barcauskaite, K., 2015. Alternative Preparation of Propolis Extracts: Comparison of Their Composition and Biological Activities., pp. 1-7, http://dx.doi.org/10.1186/s12906-015-0677-5.

Lecomte, F., Siepmann, J., Walther, M., Macrae, R.J., Bodmeier, R., 2003. Blends of enteric and GIT-insoluble polymers used for film coating: physicochemical characterization and drug release patterns. J Control. Release 89, 457-471.

Luiza, T., Oldoni, C., Cabral, I.S.R., Regitano, M.A.B., Rosalen, P.L., Ikegaki, M., Nascimento, A.M., Alencar, S.M., 2011. Isolation and analysis of bioactive isoflavonoids and chalcone from a new type of Brazilian propolis. Sep. Purif. Technol. 77, 208-213, https://doi.org/10.1016/j.seppur.2010.12.007.

Machado, B., Silva, R., Barreto, G., Costa, S., Silva, D., Brandão, H., Rocha, J., Dellagostin, O., Henriques, J., Umsza-Guez, M., Padillha, F., 2016. Chemical Composition and Biological Activity of Extracts Obtained by Supercritical Extraction and Ethanolic Extraction of Brown, Green and Red Propolis Derived from Different Geographic Regions in Brazil., pp. 1-26, http://dx.doi.org/10.1371/journal.pone.0145954.

Mahadik, K.R., Paradkar, A.R., 2015. Potentiating antimicrobial efficacy of propolis through niosomal-based system for administration. Integr. Med. Res. 4, 94-101.

Milane, A., Bezerra, F., Geyson, F., Albuquerque, F., Casimiro, G.S., Nunes, E.M., De Almeida, P.B., Sibelly, K., De Abrantes, M., De Abreu, L.C., 2015. Red Propolis Antifungal Action on Species of Candida of the Oral Cavity., pp. 1-9, http://dx.doi.org/10.3823/1735.

Nazareno, A., 2009. Chemical Variability in Propolis from Santiago del Estero, Argentina, Related to the Arboreal., pp. 978-983, http://dx.doi.org/10.1002/jsfa.3542.

Nori, M.P., Favaro-Trindade, C.S., Matias de Alencar, S., Thomazini, M., de Camargo Balieiro, J.C., Contreras Castillo, C.J., 2011. Microencapsulation of propolis extract by complex coacervation. LWT - Food Sci. Technol. 44, 429-435, https://doi.org/10.1016/j.lwt.2010.09.010.

Onbas, R., Kazan, A., Nalbantsoy, A., Yesil-celiktas, O., 2016. Cytotoxic and Nitric Oxide Inhibition Activities of Propolis Extract along with Microencapsulation by Complex Coacervation., pp. 286-293, http://dx.doi.org/10.1007/s11130-016-0558-1.

Ordó, R.M., Zampini, I.C., Moreno, M.I.N., Isla, M.I., 2011. Potential application of Northern Argentine propolis to control some phytopathogenic bacteria. Microbiol. Res. 166, 578-584, http://dx.doi.org/10.1016/j.micres.2010.11.006.

Oryan, A., Alemzadeh, E., Moshiri, A., 2018. Potential role of propolis in wound healing: biological properties and therapeutic activities. Biomed. Pharmacother. 98, 469-483, http://dx.doi.org/10.1016/j.biopha.2017.12.069.

Paulo, F., Santos, L., 2020a. New insights in the in vitro release of phenolic antioxidants: the case study of the release behavior of tyrosol from tyrosol-loaded ethylcellulose microparticles during the in vitro gastrointestinal digestion. Colloids Surf. B Biointerfaces, http://dx.doi.org/10.1016/j.colsurfb.2020.111339.

Paulo, F., Santos, L., 2020b. Encapsulation of the antioxidant tyrosol and characterization of loaded microparticles: an integrative approach on the study of the polymer carriers and loading contents. Food Bioprocess Technol. 13, 764-785, http://dx.doi.org/10.1007/s11947-020-02407-y.

Paulo, F., Santos, L., 2019. Microencapsulation of caffeic acid and its release using a w/o/w double emulsion method: assessment of formulation parameters. Dry. Technol. 37, 950-961, http://dx.doi.org/10.1080/07373937.2018.1480493.

Paulo, F., Santos, L., 2018a. Inclusion of hydroxytyrosol in ethyl cellulose microparticles: in vitro release studies under digestion conditions. Food Hydrocoll. 84, 104-116, http://dx.doi.org/10.1016/j.foodhyd.2018.06.009.

Paulo, F., Santos, L., 2018b. Double emulsion solvent evaporation approach as a novel eugenol delivery system - optimization by response surface methodology. Ind. Crop. Prod. 126, 287-301, http://dx.doi.org/10.1016/j.indcrop.2018.10.027.

Pellati, F., Prencipe, F.P., Benvenuti, S., 2013. Headspace solid-phase microextraction-gas chromatography - mass spectrometry characterization of propolis volatile compounds. J. Pharm. Biomed. Anal. 84, 103-111, http://dx.doi.org/10.1016/j.jpba.2013.05.045.

Rocha-parra, D.F., Cecilia, M., Clara, M., Chirife, J., 2016. Influence of storage conditions on phenolic compounds stability, antioxidant capacity and colour of freeze-dried encapsulated red wine. LWT - Food Sci. Technol. 70, 162-170, http://dx.doi.org/10.1016/j.lwt.2016.02.038.

Santos, M.S., Estevinho, L.M., de Carvalho, C.A.L., da Silva Conceição, A.L., de Castro Almeida, R.C., 2020. Rheological and sensorial evaluation of yogurt incorporated with red propolis. J. Food Sci. Technol. 57, 1080-1089, http://dx.doi.org/10.1007/s13197-019-04142-5.

Silva, J.C., Rodrigues, S., Feás, X., Estevinho, L.M., 2012. Antimicrobial activity, phenolic profile and role in the inflammation of propolis. Food Chem. Toxicol. 50, 1790-1795, http://dx.doi.org/10.1016/j.fct.2012.02.097.

Silva, F.C., Favaro-trindade, C.S., De Alencar, S.M., Thomazini, M., 2011. Physicochemical Properties, Antioxidant Activity and Stability of Spray-Dried Propolis., http://dx.doi.org/10.3896/IBRA.4.03.2.05.

Singleton, V.O., Rudolf Lamuela-Raventós, R., 1999. Analysis of total phenols and other oxidation substrates and antioxidants by means of Folin-Ciocalteu reagent. Oxid. Antioxid. Part A Methods Enzymol. 299, 152-178.

Valenzuela-barra, G., Castro, C., Figueroa, C., Barriga, A., Silva, X., De, B., Hortelano, S., Delporte, C., 2015. Anti-inflammatory activity and phenolic profile of propolis from two locations in Región Metropolitana de Santiago, Chile. J. Ethnopharmacol. 168, 37-44, http://dx.doi.org/10.1016/j.jep.2015.03.050.

Vyssotski, M., Lagutin, K., Catchpole, O., 2018. Simple lipids and hydrocarbons of New Zealand propolis wax. J. Apic. Res. 8839, 1-7, http://dx.doi.org/10.1080/00218839.2017.1384438.

Wu, G., Meininger, C.J., 2002. Regulation of nitric oxide synthesis by dietary factors. Annu. Rev. Nutr. 22, 61-86, http://dx.doi.org/10.1146/annurev.nutr.22.110901.145329.

Yang, S., Wu, J., Wang, Y., Huang, C., Wu, T., Shieh, C., Chang, C.J., 2014. Encapsulation of propolis flavonoids in a water soluble polymer using pressurized carbon dioxide anti-solvent crystallization. J. Supercrit. Fluids 94, 138-146, http://dx.doi.org/10.1016/j.supflu.2014.07.009.

Zabaiou, N., Fouache, A., Trousson, A., Baron, S., Zellagui, A., Lahouel, M., Lobaccaro, J.A., 2017. Biological properties of propolis extracts: something new from an ancient product. Chem. Phys. Lipids 207, 214-222, http://dx.doi.org/10.1016/j.chemphyslip.2017.04.005.

Zhang, H., Fu, Y., Niu, F., Li, Z., Ba, C., Jin, B., Chen, G., 2018. Enhanced antioxidant activity and in vitro release of propolis by acid-induced aggregation using heat-denatured zein and carboxymethyl chitosan. Food Hydrocoll. 81, 104-112, http://dx.doi.org/10.1016/j.foodhyd.2018.02.019. 